\title{
ESPECIES FUNGICAS DE CHILE: INDICE ALFABETICO
}

\author{
(Fungal species of Chile: Alphabetical index)
}

Waldo Lazo, A.

Departamento de Ciencias Ecológicas, Facultad de

Ciencias, Universidad de Chile, Santiago

Palabras clave: Especies fúngicas, indice, Chile.

Key words: Fungal species, index, Chile

\section{RESUMEN}

El presente trabajo, ain incompleto, presenta un listado de especies füngicas descritas para Chile. Se obtuvo al consultar diversas publicaciones pertinentes, muchas de las cuales se citan en las Referencias. La información micológica del pais estámuy dispersa anivel nacional e internacional y no es fácil obtenerla, por lo cual el lector encontrará algunas carencias respecto a autores, especies, sinonimias.

\section{SUMMARY}

The present paper though incomplete, includes a listing of fungal species described for Chile. They were found after looking up in specialized literature as it can be seen under References. The mycological information of the country is very much dispersed at national and international levels and it is very difficult to get it, this is why the readers will find that some data regarding authors, species, synonyms are missing. aberrans Diaporthe Speg. aberrans Microthyrium Speg. aberrans Neoclitocybe Sing. abietina Peziza Pers. ex. Fr. abnormis Peziza Mont. abnormis Puccinia P. Henn. abscondita Sphaeropsis Diet. et Neger. acaciae Pichia Van der Walt acaenae Phyllachora P. Henn. acaenae Ustilago Diet. et Neger. acanthosyrinus Crepidotus Sing. acantophilum Melanomma Speg. accentrica Fontanospora accuminatus Paneolus (Schaeff. ex Sec.) Quél. acentibularioides Geopyxis Speg. acervatum Actidium Mont. acicola Scirrhia (Dearn.) Sigger acicola Septoria (Thum.) Sacc. (= Lecanosticta acicola, = Cryptosporium acicola) acicula Mycena (Schaeff.) Sacc. aciculare Helotium Pers. acrospermum Sphaeronaema Tode actiniceps Chaetotyphula (Petch) Corner aculeifer Polystictus Berk. et Curt. acuminata Alatospora Ingold acuminata Flabellospora Descals \& Webster

acuminatum Trichophyton Sabour. acuta Clavaria Fr.

acutatum Colletotrichum Dingley \& Gilmour

acutissima Clavaria Berk. acutissima Psathyrella Sing. adamantinus Fomes Berk. adesmiae Fusarium P. Henn. adonis Camarophyllus Sing. adusta Bjerkandera (Wild. ex Fr.) Karst.(Polyporus, Polystictus) aegerita Agrocybe (Brig). Sing. aeroginosum Helotium $\mathrm{Fr}$. aestivalis Peronospora Sydow ( = P.trifoliorum De Bary) aextoxici Diaporthe Speg. aextoxici Hendersonia Speg. aextoxici Lizonia Speg. aextoxici Metasphaeria Speg. aevtoxici Phyllosticta Speg. affinis Badhamia Rost.

affinis Trichia

agaricina Exidia Mont. aggregata Torulopsis agrocybiformis Pholiota Sing. agyrothamniae Asteroma Allesch. aiacu Sphaerella Speg. ajelloi Keratinomyces (Trichophyton) (Vanbreuseghem) Ajello alacaluf Anthostoma Speg. alba Campanella (Berk et Curt.) Sing. alba Cyttaria Phil.

alba Mikronegeria Oehrens et Peterson albicans Candida (Robin) Berkhout albicens Cytospora Ell. et Kellerm. albida Puccinia Diet et Neger albida Tremella Huds. albido-rosea Trametes Bomm. \& Rouss. albidobisa Clavaria Sing, albidum Scleroderma Pat. \& Trab. emend. Guzmán albidus Agaricus Pers. albidus Dacryomyces Bert. albo-atrum Verticillium Reinke et Berth.

albocinctus Cortinarius Moser albogrisea Mycena Pcck albomaculata Xylaria album Arachnion Schw. album Diplosporium Bon. album Trichophyton Sabouraud. (=T.verrucosum Bodin) albus Polyporus (Huds.) Fr. 
aleuriatus Bolbitius (Fr.ex Fr.)Sing. alfalfae Urophlycts (Lagh.)Magn. algerinensis Mycena Maire. allii Puccinia (DC.) Rud. alneum Schizophyllm Schroet. alni Microsphaera (Wallr.) Lev. alopecuri Uromyces Speg. alpina Guepiniopsis (Tracy \& Earle) Brasf.

alsinearum Peronospora Casp. alstroemeriaeAecidium Diet. et Neger alstroemeriae Hendersonulina Speg. alstroemeriae Pleospora Speg. alstroemeriae Porterula Speg. alstroemeriae Puccinia Syd alstroemeriae Sphaerella Speg. alstroemeriae Uredo Dict. alstroemeriae Uromyces (Dict.) P, Henn.

alternata Alternaria (Fr.) Kreissl. alveolataArcangeliella (Ckc.\& Mass) Zeller \& Dodge

alveolata Martelia (Cke. et Mass) A.H.Smith

allantosporum Anthostoma Spcg. alliaceus Aspergillus Thom \& Church alli Aecidium Pers.

allii Botrytis Mann.

allii Helminthosporium Campanile. (=Embellisia allii (Camp.) Simmons )

allii Heterosporium Ell. \& Mart. allii-populina Melampsora Klcb. allii-sativi Fusarium Allesch. alliodorus Marasmiellus (Bert.ex Mont.) Sing. (Marasmius, CanthareHus)

allocy'stis Agrocybe Sing. amadelpha Cercospora Sỵd. amara Torula amarissimus Gymmopilus Murr. ambigua Puccinia (Alb.\& Sclıw.) Lagerth

ambrosiae Oidium Thucm. americana Pestalotia Mont. (= Harknesia americana (Mont.) Sulton americana Uncinula Howc. americanus Uromy'ces Speg. amianthinum Cystoderma (Scot.) Fr. ammophila Sarcosphaera (Dur. \& Lé:) Mocz.

amoena Clavulinopsis (Zoll. \& Mar.) Corner ampelina Elsinoë (D'By.) Shear. ampelinum Sphaceloma D'By, ampelophagum Gloeosporium (Pass.) Sacc.

amphitricha Meliola Fr.

amygdali Fusicladium Ducom. amygdalina Mycena (Pers.) Sing. anamorph Malbranchea de Uncinocarpus reesii Sigler \& Orr

ancudense Aecidium Speg. andicola Kuehneola Diet. andicola Uredo Dict. \& Neger andina Arnaudiella Butin \& Peredo andina Phoma Sacc. \& Syd. andina Puccinia Diet. \& Neger andinus Uromyces Magn. andropogonis Puccinia Scliw. andropogonis Uromyces Tracy androsaceus Marasmius (L.) Fr. anemone Urocy'stis (Pers.) Schroet. anemone Ustilago (Pers.) Schroct.

angulata Articulospora Tubaki angulata Psilocybe (Pers) Sing. angulata Puccinia Dict. \& Neger angulatum Tricladium Ingold angulatus Marasmius (Pers.) Berk. angustata Sphaeria Pers. annelidicu Filosporella (Shcarer \& Cranc ) Crane \& Slicarer annonae Phomopsis De Urrie. annularis Puccinia (Strauss) Schlecht. anmulata Stictis Ckc. \& Phil. annulatum Hyposylon (Schw.) Mont. annuแm Fusarium Lconian. anomala Peziza Pers. anomala Puccinia Rost. anomalus Marasmius Lasch.in Rab. antarctica Bovista Speg antarctica Calosphaeria Speg. antarctica Coprolepa Speg. (Hypocopra) antarctica Coryneliopsis Butin. antarctica Crumenula Rchm antarctica Cucurbitaria Speg. antarctica Cumminsiella (Speg.) Baxter

antarctica Cunninghamella Caretta $\&$ Piontelli antarctica Cytospora Speg. antarctica Diderma (Spcg.) Sturgis. antarctica Dothidea Speg. antarctica Fistulina Spcg antarctica Galerina Sing. antarctica Ciloniella Speg. antarctica Gjromitra Rehm. antarctica Harknessia Speg. antarctica Heterobotrys Speg. antarctica Hirneola Speg. antarctica Humaria Speg. antarctica Hypocopra Speg. antarctica Laboulbenia Speg. antarctica Licea Speg. antarctica Melanconis Speg. antarctica Melanospora Speg. antarctica Mitrula Speg. antarctica Morenoella Speg. antarctica Omphalina Sing. antarctica Peziza Speg. antarctica Phoma Speg. antarctica Phyllactinia Speg, antarctica Puccinia Speg. antarctica Pyrenophora Speg. antarctica Rhamphoria Speg. antarctica Selinia Speg. antarctica Septoria Speg. antarctica Sphaerella Speg. antarctica Sporocybe Speg. antarctica Tympanis Speg antarctica Xylaria Speg. antarcticum Corticium Speg. antarcticum Enteridium Speg. antarcticum Exobasidium Speg. antarcticum Lecanidium Speg. . antarcticum Lophiotrema Speg antharcticus Agyrium Rchm. anfarcticus Marasmicllus Sing anfarcticus Ophiobolus Speg. antarcticus Polystictus Speg. antherarum Ustilago $\mathrm{Fr}$. anthoxanthina Puccinia (Bub.)Gaum. anthracodes Hypoxylon (Fr.) Mont. anthracoides Sphacria Fr. anthracophila Peziza Dennis anthirrini Suptoria Desm. antillancac Entoloma Horak antarcticum Lophodermium Spćg. antucensis Puccinia Syd. aphanidermatum Py rhium(Eds) Fit/p. apiculata Entomophthora (Thasi) Gustavson apiculata Kloceker. (Rus Everh. Klöcker) Janje apii Septoria (Br. \& Cav:) Chester. apii Puccinia Cda. apii-graveolantis Septoria Dor. apiicola Septoria Speg. apiospora Splhaeria Dur. et Mont. apora Psathyrella Sing. aporpohyphes Marasmius Sing. 
aporpus Marasmius Sing.

appendiculata Halosphaeria Linder appendiculatus Hypholoma Bull. (Agaricus)

appendiculatus Uromyces Fr. applanata Sphaeria Fr.

applanatus Crepidotus (Pers. ex Fr.) Kummer

applanatus Fomes (Pers. ex Wallr.) Gill.

applicatus Pleurotus (Batsch.) Fr. (Agaricus)

applicatus Resupinatus (Batsch. ex Fr.) S.F Gray

apus Puccinia Cda.

aquatica Bacillispora Dor.

aquatica Clavariopsis De Wildeman aquatica Culicidospora Petersen aquatica Lemonniera De Wildeman aquatica Margaritispora Ingold aquatica Pyricularia Ingold aquosus Mucor Mart. araucana Eutryblidiella Butin araucana Gloniella Speg. araucana Gloniopsis Speg. araucana Hygrocybe Sing. araucana Microthelia Speg. araucana Phoma Speg. araucana Puccinia Diet. \& Neger araucana Pyrenopeziza Butin araucanum Acremonium Speg. araucanum Glonium Speg. araucanus Uromyces Diet. \& Neger araucariae Atopospora Butin araucariae Ceratocystis Butin araucariae Phaeocryptopus Butin araucariae Rhodotorula Grinbegs et Yarrow.

arctica Pleospora Karst. arenariae Puccinia Wint. arenarius Polyporus K1. arenicola Sepultaria (Lev.) Massee. arenosa Lachnea (Fckl.) Gillet. areolatum Physarum Bert. argentea Lycogala Pers. argentina Psilocybe (Speg.) Sing. argillacea Geosmithia Pitt arguillaceum Fusisporium Fr. aridum Corticium Fr. aridum Aecidium Diet. et Neger aridus Cortinarius Moser aristidae Placosphaeria Allesch. aristidae Puccinia Tracy aristidae Sorospodium Neger aristotelia Mycenella Sing. aristoteliae Crepidotus Sing. armeriae Uredo Duby. armeriae Uromyces Lév. aromatica Botryodiplodia Speg. artocreas Discosia (Tode) Fr. arundinaca Puccinia Hedw. arundinaceum Lophodermium (Schrad) Chev. arvensis Agaricus Schaeff. ascoboloides Peziza Bert. ascophora Pholiota (Peck) Sing. asiatica Septoria Speg. aspera Bovistella Lév. aspera Bovista Lév. asperella Lamprospora (Rehm) Boud. asperothecum Helicoma Linder asperum Lycoperdon (Lév.) Speg. asteliae Galerina Sing. astericina Diaporthe Speg. asterisci Hendersonulina asterisci Phyllosticta Speg. astomum Microthyrium Speg. astragali Uredo Opiz. atacamense Caeoma Syd. atacammae Systremma Syd. aterrima Ombrophila Mass. atomatus Paneolus Fr. atra Chaetomella Fuckel. atra Stachybotrys Cda. atramentarius Coprinus (Bull.) Fr. atrata Peziza Pers.

atriavellaneus Pluteus Sing. atrifolia Psathyrella (Peck) A.H. Smith atrofibrillosus Leucoagaricus Sing. atrofuligineus Agaricus Bert. atropungens Pluteus Smithet Bertelli. atrorubens Marasmius Berk. atroseptatum Helicoma Linder atrovirens Hypocrea Mont. atrovirens Spondylocladium Hazz.ex Sacc. atrum Ulocladium Preuss. auctum Lophiotrema Sacey. audouini Microsporum Gruby. augustatum Hysterium Alb. et Schw. augustus AgaricusFr.

aulaxina Favolaschia (Mont.) Garrido aulaxinus Agaricus Mont aurantia Aleuria (Pers. ex Fr.) Fuckel (Peziza) aurantiaca Cribraria Schrad. aurantiaca Gymnascella Peck aurantiaca Hygrophoropsis (Wulf. ex

Fr.) Maire (Agaricus)

aurantiacus Thermoascus Miche

aurantiovelata Amanita Schalvick. et Jansen.

aurantius Hypomyces (Pers.) Fckl.

aurantitus Uromyces

aurea Clavaria Schaeff.

aurea Taphrina (Pers.) Fr.

aureum Physarum Pers.

aureum Synchytrium Schroet.

aureus Amauroascus (Eidam)y. Arx

aureus Polyporus

auricula-judae Exidia Fr.

aurivella Pholiota (Batsch ex Fr.)

Kummer

aeruginosum Chlorosplenium (Oed.)

de Not.

australe Cenangium Speg.

australe Ceratostoma Speg.

australe Ganoderma (Fr.) Pat.

(Polyporus)

australe Melanomma Speg.

australiensis Leptosphaeria(Cribb. \& Cribb.) G. Hughes

australis Ameghiniella Speg.

australis Blysporus Fr.(Ganoderma)

australis Darluca (Biv.) Cast.

australis Milesia Arth.

austratis Milesina (Arth.) Hiratsuka

australis Nectria Mont.

australis Nidularia Tul.

australis Phaeocryptopus Butin.

australis Pholiotina Sing.

australis Ravenelia Diet. et Neger

australis Rhodocybe Sing.

austrulis Uredo Diet. et Neger

austroacutus Cortinarius Moser

austroanatinum Entoloma (Singer)

Horak (Rhodophyllus)

austroandina Xeromphalina Kühner

$\&$ Maire

austroandinus Crepidotus Sing.

austroantarcticus Rhodophyllus Sing. austrocalyptrata Galerina Sing. austrocapillaris Mycena Sing. austrocedri Appendiculella Butin austrochilensis Campanella Sing. austrochilensis Micromphale Sing. austrodelica Russula Sing. austrofallax Cystoderma Sing. austrolimonius Cortinarius Moser et Horak

austrosalor Cortinarius Moser 
austrotororida Mycena Sing. austrotorula Marasmius Sing. austroturmalis Cortinarius Moser autralis Seynesia Speg. autummalis Meliola Syd. avenae Macrosporium Oud. avenae Ustilago (Pers) Rost. axifera Stemonites (Bull.) $\mathrm{Mc} \mathrm{Br}$. azarae Asterina Lév. azarae Uredo Neger. azureus Polystictus Fr. azureus Stephanopus Moser \& Horak.

babingtonii Psilopezia (Berk. \& Br.) Berk.

baccharidicola Septoria Speg. baccharidiphila Sphaerella Speg. baccharidis Caeoma (Lév.) Diet. et Neger

baccharidis Coleosporium (Lév.) Cke. baccharidis Eriosporangium Bert. baccharidis Puccinia Diet. et Holw. baccharidis Sphaeropsis (Allesch.) Bert.

baccharidis Uredo Lév. badia Peziza Pers.

badio-confusa Peziza Korf. baeosperma Pholiota Sing. baeumleri Microsphaera Magn. bagnisiana Durella Sacc. barba-jovis Odontia (With.)Fr. barda Torrubiella Petch. barri-aranae Puccinia Diet. et Neger bassiana Botrytis Bals. batucense Hysterium Speg. bedellomorphum Helminthosporium Speg.

beijerinckii Coryneum Oud. beijerinckii Stereum bellotae Venturia Speg. beniensis Phaeogyroporus Speg. berberidicola Pleurotus sensu. Sing. (Agaricus) berberidincola Amphisphaeria Rehm. berberidis Aecidium Gmel. berberidis Caeoma Har. berberidis Curreya Rehm. berberidis Epitea Lév. berberidis Lophodermium (Schl.) Rehm.

berberidis Plowrightia (Wallr.) Sacc. berberidis Puccinia Mont. berberidis Uredo Lév. berterii Anthracophyllum (Mont)
Sing.

berterii Hypoxylon Mont.

berterii Mitrula Mont.

berterii Sphaeria Mont.

berterii Xerotus Mont.

berteroanum Schizostoma (Mont.) Sing.

berteroi Cyttaria Berk.

betae Phoma Fr.

betae Septoria West.

betae Uredo Pers.

betae Uromyces (Pers.) Lév.

berteroana Licea Mont.

bethelii Plyssarum $\mathrm{Mc} \mathrm{Br}$. ex Lister

beticola Cercospora Sacc.

betulina Lenzites (L) Fr. (Polyporus)

bicolor Dasyscyphus (Bull.ex Fr.)

Fuck.

bicolor Hydnum Alb. ex Schw.

bicolor Peziza Bull.

bidenticola Uromyces (P.Henn.) Arth. bidwelli Guignardia (EII.) Viala \& Ravar

biennis Polyporus (Bull) Fr.

biformis Phyllosticta Heald et Wold.

biformis Sphaeria Pers.

bigelowii Melampsora Thuem.

biocellatum Phoma Mont.

bisporula Trichodelitschia (Cr.) Munk.

bisporus Agaricus (Lange) Pilát

biturbinata Pontoporeia $(=$ Zopfia $)$

bivalve Physarum Pers.

blechni Milesia (Syd.) Arth.

blechni Uredo Diet. et Neger

blechnii Pholiota Sing.

blechnophila Mycena Sing.

bliti Albugo (Biv. Bern) Kunze

blitis Cystopus (Biv.) Duby

boldoae Coniothyrium Speg.

boldoae Didymosphaeria Speg.

boldoae Diplodia Speg.

boldoae Ellisiella Speg.

boldoae Herpotrichia Speg.

boldoae Meliolopsis Speg.

boldoae Phoma Speg.

boldoae Pleospora Speg.

boldoae Valsonectria Speg.

boldoicola Phoma Speg.

boletinoides Parapaxillus Sing.

boletinoides Paxillus Sing.

bomareae Urocystis Diet. et Neger

bombarda Sphaeria Batsch

bombycinum Helminthosporium
Walh.

bonaerensis Macrolepiota (Speg.)

Sing. (Agaricus)

bonariensis Uredo Speg.

boopis Puccinia Neger

boquilae Sphaerella Speg.

botryosum Stemphylium Wallr.

botrytis Trichia Pers.

boudieri Coprinus Quél.

bovei Hypoxylon Speg.

bovinoides Cortinarius Moser \& Horak.

bovista Arachnion Mont.

bovista Lycoperdon L,

bovista Scleroderma Fr.

brachypodii Puccinia Atth.

brachysporum Hypoderma Speg.

brasiliensis Tremella (Moller) Lloyd

brassicae Alternaria (Berk.) Sacc.

brassicae Cercosporella (Fautr. et

Roum.)V. Hön.

brassicae Phyllosticta (Curr.) West. brassicae Plasmodiophora Wallr.

brassisicola Alternaria (Berk.) Sacc.

brevicompactum Penicillium

Dierckx

brevipes Caliciopsis Butin

brevisporus Cortinarius Moser

bridgesii Phyllosticta Speg.

briggesiana Inocybe

bromi Phyllachora Fuckel

bromicola Septoria Speg.

bromicola Uromyces Arth. \& Howol.

bromivora Ustilago (Tul.) E. Fisch

brownii Mycena Smith

bruchii Melanotus (Speg.) Horak

(Crepidotus)

brumptii Candida Lang. \& Guerra.

bruneolum Physarum (Phil.) Mass.

brunnea Bovistella

brunneoolivaceus Pluteus

brunneorugosus Cortinarius Moser

brunneovelatus Cortinarius Moser \& Horak

brunneum Entoloma Horak

brunswickianus Crepidotus (Speg.)

Sing, (Agaricus)

bryophilus Merulius Pers.

buccina Helotium (Pers.) Fr.

buddleiae Septoria Kalchb.

bulbifaciens Aecidium Neger

bulbigerus Pluteus Horak

bulbillosus Coprinus Pat.

bulbipes Marasmius Mont. 
bulbosomustellinus Cortinarius Moser \& Horak

bullata Taphrina Tul.

bullata Ustilago Berk.

bullatus Exoascus (Berk. exFr.)Fuck.

bullulifer Crepidotus Sing.

bunsteri Aecidium Neger

bunsteri Uromyces (Neger) Jacks. \& Howl.

bursaeformis Hohenbuehelia (Berk.) Reid (Agaricus, Conchomyces)

butyracea, Collybia (Bull. ex Fr.)

Kummer (Agaricus)

byssiseda Neoclitocybe (Bres.) Sing. (Omphalia)

cactorum Phytophthora (Leb. \& Cohn.) Schroet.

caelata Rhodocybe (Fr.) R. Maire (Agaricus)

caelatum Lycoperdon Bull. caesia Mollisia (Fuck.) Sacc. caesioanulata Psilocybe Sing. caesiocana Mycena Sing.

caesium Agyrium Fr. caespitulosum Septobasidium Bres. calceum Corticium (Pers.) Fr. caldcluviae Metasphaeria Speg. calendulae Entyloma (Oud.) D'By californiense Auxarthron Orr \& Kuehn

calycerae Puccinia Syd. calyculata Hemitrichia (Speg.) Farr callaquensis Puccinia Neger. callosa Psilocybe (Fr.ex Fr.) Quél. (Agaricus)

camelliae Phyllosticta West. campanella Agaricus Batsch. campanulata Nidularia Fr. campanulatus Paneolus L. campestris Agaricus L. (Psalliota) campoi Clavaria Speg. campoi Cortinarius Speg. campoi Nidularia Speg. campoi Panus Speg. campoi Pterula Speg. campoi Septoria Speg. campoi Sphaerella Speg. camptophylla Mycena (Berk.)Sing. (Agaricus)

cancellatus Dictydium (Batsch) Mc Bride.

candida Albugo (Pers.)Kuntze. candida Himantia (Hude)Pers. candida Thelephora Schw. candida Torulopsis (Saito)Loder. candidissimun Cheimonophyllum (Berk, ex Br.) Sing.(Agaricus) candidum Geotrichum Link. candidus Aspergillus Link. candidus Cystopus (Pers.)Lév candidus Dacryomyces candolleana Psathyrella

Maire. (Agaricus)

canis Microsporum Bodin capillaris Mycena (Schum. ex Fr.)

Kummer (Agaricus)

capillipes Marasmius Sacc.

capitatum Physarum Link. capnoides Naematoloma (Fr. ex Fr.) Karst. (Agaricus, Hypholoma) capsularis Endomycopsis (Schiönnings) Dekk.

capsulatum Histoplasma Darling. capsulifera Badhamia (Bull.)Berk. capula Cyphella P. Henn. caracolensis Cytospora Speg. carbo Ustilago Tul. carbo Uredo DC. carbonaria Pholiota(Fr.) Sing. (Agaricus)

carbonaria Psilocybe Sing. cardamine-cordatae Puccinia Diet. \& Neger cardinale Coryneum Wagener ( = Seiridium cardinale (Wagen.) Sutton \& Gibson)

caricae Pucciniopsis (Speg.) Earle caricina Puccinia DC. caricis Anthracoidea (Pers.) Bref. caricis Puccinia (Schum.) Reb. caricis-bracteosae Puccinia Speg. caricis-haematorhynchae Puccinia Diet. \&. Neger

caries Tilletia (DC.)Tul.

caries Uredo DC.

carmichaelii Chrysosporium Van Oorschot carneolus Cortinarius Moser et Horak carneoroseum Thaxterogaster Horak curneus Aspergillus Blochwitz carneus Iodophanus (Pers.) Korf. carotae Cercospora (Pass.)Sohl. carpophilum Cladosporium Thüm. carpophilum Clasterosporium (Lev) Aderh.

carpophillum Fusicladium (Thüm.) Aderh. carpophyllum Coryneum (Lév.) Jauch. carthamis Puccinia Hutzl.

caryiophyllacearum Melampsorella Schroet.

caryophylaceus Polyporus

caryophyllinus Uromyces (Schrank.)

Wint.

casearius Polyporus Fr.

cashiae Lamprospora Gamundi

cassiaecolor Melanotus (Berk.)Sing.

(Agaricus)

castagnei Sphaerotheca Lév.

castaneiceps Kuehneromyces

catillus Exidia Mont.

caulicola Peziza Fr.

cochlearis Caliciopsis Butin

cyathus Cyphella P. Henn.

cenchri Puccinia Diet. \& Holw.

cenereus Thecotheus $(\mathrm{Cr}$. et $\mathrm{Cr}$.)

Chenantais

centrifugum Corticium (Lév.) Bres.

cepa Sclerotinia

cepa Scleroderma Pers.

cepae Sclerotium Berk. et Br.

cepivorum Sclerotium Berk.

cepulae Urocystis Forst.

cerasi Exoascus Fckl.

cerasi Taphrina Sadeb.

cerasphora Astrosporina (Singer)

Horak (Inoscybe)

cerastii Septoria Rob. \& Desm.

cerebella Coniophora (Pers.) Duby. cereicola Pleospora Speg.

cerina Dasyscypha (Pers.) Fuckel.

$$
\text { (Peziza) }
$$

cervina Bovista Berk.

cervinus Elaphomyces (L.) Fr.

cervinus Pluteus (Schaeff.) Fr.

cestri Aecidium Mont.

cestri Puccinia Bert.

cestri Septoria Mont.

cestri Sphaeria Bert.

cestri Uredo Bert.

cestri Uromyces Mont

$c h . .$. . después de $c y .$. (after $c y . .$. )

cibarius Clathrus (Tul.)E. Fisch.

cichoracearum Erysiphe DC,

cichoracearum Uromyces Fr.

cichorii Puccinia (DC.) Bellynck.

ciliata Discocyphella P. Henn.

ciliata Gloiocephala (Henn.) Sing.

ciliatus Lasiobolus (Schmidt. exFr.)

Boud.

ciliatus Phaeomarasmius Sing.

cilotus Aguricus Speg. (Mycena) 
cinerariae Alternaria Hori. \& Enj. cinerea Arcyria (Bull.)Fr.

cinerea Botrytis Pers. cinerea Mollisia (Batsch)Karst. cinerea Monilia Bon. cinereum Physarum (Batsch) Pers. cingens Puccinia Bomm. \& Rouss. cingulatum Gloeosporium Atk. cinnabarina Nectria (Tode)Fr. cinnabarina Peziza Bert. cinnabarinus Polystictus (Jacq.)Fr. cinnamomi Phytophthora Rands. cinnamonea Odontia Mont. cinnamoneum Septobasidium Burt. cinnamophyllus Cortinarius Moser. circinans Colletotrichum (Berk.) Vogl.

circinelloides Mucor Vt. circostomum Hypoxylon Speg. circumscribens Aecidium Neger circumscriptus Uromyces Neger circunscisum Catastoma (Bcrk. \& Curt.) Morgan cirsii-lanceolati Puccinia Schroct. cistophilum Hysterangium (Tul.)

Zeller \& Dodge

citri Alternaria Pierce.

citri Cladosporium Mass.

citri Diaporthe (Faw.) Wolf,

citri Limacinia

citri Phomopsis Faw.

citri Septoria Pass.

citricola Diplodia Mc. Alp.

citrina Calycella (Hedwig) Bond.

citrina Gymmascella (Massee \& Salm.)

Orr. Ghosh \& Roy

citrina Peziza Batsch

citrinella Mycena (Pers. ex Fr.) Quél. citrinopigmentosus Cortinarius Moser citrinum Helotium (Hedw.)Fr. citrinum Penicillium Thom. citrinum Phy'sarum Schum. citrinum Saccobolus Boud. et Torrend citrophthora Phytophthora Sm. \& $\mathrm{Sm}$. Leonian citrophthora Pythiacystis Sm. et. Sm. cladosporioides Cladosporium (Fr.) de Vries

cladosporioides Cercospora clarionae Puccinia Dict. \& Neger clarioneicola Puccinia Speg. clathroides Hysterangium Vitt. clathroides Hericium (Pall. ex Fr.) Pers es Pcrs. clausa Aposphaeria (Wint.) Speg. clausa Phoma Wint. clavariiformis Phyllactinia Neger clavata Caliciopsis (Lév.) Fitz. clavata Corynelia (L.) Sacc. clavata Hemiarcyria (Pers.) Rost. clavata Hemitrichia (Pers.) Rost clavata Trichia Pers.

clavatus Sphaeronaema Lév. clavatus Uromyces Dict. claviceps Balansia Speg. clavuligerum Helotium Speg. clavus Sclerotium DC. clematidis Puccinia (DC.) Lagerh. clementi Didymosphaeria Sacc. cnici Puccinia Martius. coartatus Cortinarius Horak. coccinea Hygrocybe (Fr.)Kummer (Agaricus)

coccinea Nectria (Pers.) Fr. coccinea Sphaeria Pers. coccinea Sarcoscypha (Jacq. ex $\mathrm{Fr}_{+}$) Lambotte (Peziza, Humaria) coccincum Hypoxylon Bull. cocoicola Phyllachora P.Henn. cochlearis Caliciopsis Butin cochlioides Aphanomyces Dresch. codeiophorum Tricholoma (Mont.)

Sacc.

codiophorus Agaricus Mont. colonorchidis Septoria P. Henn. coelopus Peziza Mont. coeruleum Melittosporium Rchm. cofferata Galiella Gamundi cognata Paccinia Syd. coleopus Cortinarius Moser \& Horak coliforme Myriostoma (Dicks. cx Pcrs.) Corda.

colorata Bovista (Pcck) Kreis. columbinus Cortinarius Moser \& Horak

colummatus Clathrus Bosc.

colummatus Geaster Lév:

collariata Rozites (Horak \& Moscr) Horak

colletiae Montagnella Speg. collomiae Clathrospora Rchm. collybiodes Rhodocybe Sing. in Sing \& Digilio comatus Coprinus Fr. (Agaricus) commixta Crinipellis Sing. commune Schizophyllum Fr. communis Erysiphe (Wallr.)Lind. compacta Asterina Lci: compacta Meliola (Lév.) Speg. compernis Flammula Fr. complicatum Stereum $\mathrm{Fr}$. compositarum Puccinia Schlecht. compositarum Uredo Schlecht. compressum Physarum Alb.\& Schw compresum Lophidium (Pers.) Sacc. comta Septoria Sacc.

concentrica Daldinia (Bolt.) Ces. et

De Not. (Hypoxylon)

conceptionis Pestalotia Speg. concrescens Polyporus Mont. concursa Xylaria

conferta Peronospora (Ung.) Gaum. confluens Gymmascella ( Sartory \& Bainier) Currah

confragosuliformis Phueomarasmius Sing.

conglutinans Fusarium Beach.

conglutinatus Agaricus Bert conica Morchella Pers.

conica Hygrocy (Scop, ex Fr.) Karst. (Agaricus)

conicum Sphaeronaema (Tode) Sacc. conigenum Lophpdermium (Brun.)

Hilitz

coniferatum Massarina Butin conjugatum Auxarthron (Kuchn) Orr \& Kuclın

conspurcata Dothidea Berk. constricta Alatospora Dyko contorta Trichia (Ditm.) Rost, contraversus Lactarius (Pers, ex Fr.) Fr. (Agaricus) convexulus Pancolus Sing. convolvali Septoria Desm. comyzella Puccinia Syd. cookeana Meliola Speg. cookei Microsporum Ajcllo coprinaria (heilymenia (Ckc.) Bond copriniformis Mycena Speg. coprogena Podospora Speg. coprophila Psilocybe (Bull. ex Fr.)

Kummer (Deconica) coprophila Podospora (Fr.) Ces. coquimbensis Galerina Sing. corallina Dothide'a Mont. corallina Meliola Mont. coralloides Clavaria L. coriandri Cercospora Jac\%. coriariae Didymella Speg. coriariac Phoma Speg. coriariae Rhabdospora Speg. coriariicola Phomopsis Speg. 
coriariicola Phyllosticta Speg. corium Merulius Fr.

corium Myscenastrum (Guers.) Grev. cornea Calocera (Batsch) Fr. coronata Puccinia Corda. coronilla Stropharia (Fr.) Quél. (Agaricus)

coronifera Puccinia Kleb. corralensis Lambottiela Speg. corralensis Venturia Speg. corralina Asteroma Mont. corticalis Agaricus Bert. corticalis Perichaena (Batsch) Rost. corticis Kloeckera (Klöcker) Janke corticis Sphaeria Fr. corticola Myxosporium Edg. corticola Pezicula (Jorgenssen) Nannf. cortinatellum Tricholoma Sing. cortinatum Tricholoma Sing. corymbosa Ascoidea Gams. \& Grinberg corymbosus Mucor Wallr. costatum Didymium Fr. costesi Capnodinula Speg. costesi Chaetodiplodia Speg. costesi Ectosphaeria Speg. costesi Glonium Speg. costesi Metasphaeria Speg. costesi Phyllosticta Speg. costesi Rosellinia Speg. costesi Xerulina (Speg.) Sing. costesi Zukalia Speg. costesii Cyptotrama (Speg.) Sing costesii Xerulina Speg: costesianus Uromyces Speg. couleu Marasmiellus Sing. crassa Anguillospora Ingold crassa Mollisia Boud. crassa Sphaerella Awers. crassipes Guepinia Mont. crassipes Uromyces Diet. \& Neger crassispora Septoria Wint. crassivela Pholiota Speg. creoleucum Hypoxylon Speg. crepini Leptosphaeria (Westend.) De N.

cressae Aecidium DC. cressae Puccinia Lagh. crinodendri Phyllosticta Speg. crispata Mycena (Kühn) Sing cristata Corallospora (Kohlm.) Kohlm.

cristata Lepiota (Alb. ex Fr.) Kummm (Agaricus) cristata Hemimycena (Kühner) Sing. crocatum Hypoxylon Mont. croceo-sanguinea Pleuroflammula (Mont.) Sing. (Agaricus) crucibulum Cyathus Pers. crukshanksiae Uromyces Cumm. \& Bonnard

crustacea Mucilago Wiggers crustaceum Penicillium (L.) Fr. crustuliniforme Hebeloma (Bull. ex Fr.) Quél.(Agaricus) cryptica Puccinia Arth. \& Howl. cryptochroum Entoloma (Sing.) Horak (Rhodophyllus) cryptocystis Conocybe (Atk.) Sing. (Galerula)

crystallinus Pilobolus (Wigg.) Tode crystallophorus Cortinarius Moser \& Horak

crythroscus Agaricus Pers.

cucumerinum Cladosporium Ell. \& Arth.

cucumerina Alternaria (Ell. \& Ev.) J.A Elliot

cucumis Macrocystidia (Pers. ex Fr.) Hein.

cucurbita Entoloma (Rhodophyllus) Horak

cucurbitacearum Sphaeria Fr.

culcitii Puccinia Syd.

culmicola Leptosphaeria (Fr.) Karst.

culmicola Pleospora Speg.

culmigenum Coniosporium (Berk.) Sacc.

culmiphraga Leptosphaeria $(\mathrm{Fr}$ ) Ces. et de Not.

cumingi Leptostroma Speg. cummingi Cryptosphaerina Speg. cummingii Glonium Speg. cummingii Hysterographium Speg. cunctabundus Coprinus Mont. cupulifera Coryneliopsis Butin curicoana Puccinia Syd. curvula Flagellospora Ingold curvula Lunuluspora Ingold curvula Pleurage (D'By.) Kuntze (=Schizotecium) cuspidatus Uromyces Wint. cutaneum Trichosporum (De Beurm, Gougerot. \& Vaucher) Ota. cuzcoensis Puccinia Arth. cyanella Mycena Sing. cyanocephalus Basidopus (Sing.) Horak (Mycena) cyathiformis Calvatia (Bosc.) Morgan cycliscus Polyporus Mont. cyclodes Polystictus Fr. cyclopium Penicillium Westling cyclostoma Caeoma (Lév.) Hart. cyclostoma Uredo Lév. cyclostoma Uromyces Lév. cylindrica Agrocybe (Fr.) Maire (Agaricus)

cylindrosporum Hysterographium Rehm.

cylindrotheca Asterinella (Speg.) Th. cymbisperma Anthostomella Wint. cynapii Uredo DC.

cynarae Ascochyta Maf. cynarae Ramularia Sacc. cynoctoni Puccinia Lév. cynodontis Helminthosporium Marig. cynodontois Puccinia Desm. cystidiosus Kuehneromyces Sing. cythoides Phialea (Bull.) Gill. cyttariae Chalara Bom. \& Rouss. cyttariarum Ophiostomella Butin \& von Arx chaetantherae Uredo Neger. chaetocladia Campylospora chamaelea Peziza Bert. chartarum Alternaria Preuss. chascolytri Uredo Diet. \& Neger. chechqueraultii Lamprospora (Grouan.) Boud. chenopodi Peronospora Schlecht. cherimoliae Ascochyta Thuem. chilense Agyrium Speg. chilense Actinothecium Speg: chilense Acremonium Morgan-Jones, White \& Piontelli chilense Camarosporulum Speg. chilense Eurotium Mont. chilense Lycoperdon Speg. chilense Lophiotrema Speg. chilense Oidium Linder chilense Mycenastrum Mont. chilense Trichothyrium Speg. chilensis Apiospora Speg. chilensis Arthrobothrys Allesch. P. Henn. chilensis Asterotus chilensis Atichia Cotton chilensis Belonium Speg. chilensis Boletus Sing. chilensis Brefeldiela Speg. chilensis Bulgaria Mont. chilensis Candida Grinbergs. \& Yarrow. chilensis Catharinia Speg. 
chilensis Claudopus Speg. chilensis Coccobotrys Speg. chilensis Clypeosphaeria Speg.

chilensis Crepidotus (Agaricus) Horak.

chilensis Cryptospora Speg. chilensis Cryptovalsa Speg. chilensis Cytispora Mont. chilensis Diatrype P. Henn. chilensis Dilophospora Speg. chilensis Diplodina Speg. chilensis Dothiorella Speg. chilensis Elsinoë Bit. chilensis Ellisiella Speg. chilensis Eutypella Speg. chilensis Fabraea Speg. chilensis Fomes Fr. chilensis Fusisporium Mont. chilensis Gautieria Zeller, et Dodge chilensis Gloniella Speg. chilensis Glonium Speg. chilensis Gymnopilus Sing. chilensis Heptameria Speg. chilensis Herpotrichia Speg. chilensis Heteropetella Speg. chilensis Hygrocybe Sing. chilensis Hysterium Speg. chilensis Inocybe Speg. chilensis Isothea Speg. chilensis Jaffuela Speg. chilensis Kalmusia Speg. chilensis Laboulbenia Speg. chilensis Laccaria (Mont.) Sing. chilensis Lambottiela Speg. chilensis Lepidonectria Speg. chilensis Leptosphaeria Speg. chilensis Lophidiopsis Speg. chilensis Marasmiellus (Speg.) Sing. chilensis Massarinula Speg chilensis Melanomma Speg.. chilensis Melanopsamma Speg. chilensis Meliola Speg. chilensis Melomastia Speg. chilensis Merulius Speg. chilensis Miriophysella Speg. chilensis Mitopeltis Speg. chilensis Montagnella Speg. chilensis Myriophysella Speg. chilensis Negeriella P. Henn. chilensis Niptera Speg. chilensis Octavianina Horak chilensis Ombrophila (Mont) Sacc. chilensis Omphalina (Mont.) Sing. chilensis Ophiobolus Speg. chilensis Pellicularia Speg. chilensis Phyllosticta Allesch. chilensis Physalosporella Speg. chilensis Plectania (Mont.) Gamundí chilensis Psathyrella Speg. chilensis Pseudobaeospora Horak chilensis Psilocybe Sing. chilensis Puccinia Diet. et Neger chilensis Pyrenopeziza Speg. chilensis Quaternaria Speg. chilensis Resupinatus Sing. chilensis Rhodogaster Horak. chilensis Sarcosoma (Mont.) Le Gal chilensis Seynesia Speg. chilensis Speira Speg. chilensis Sphaeronemopsis Speg. chilensis Stictis Speg.

chilensis Stomiopeltis chilensis Stropharia Speg. chilensis Trochila Speg. chilensis Trotterula Speg. chilensis Uleiella Diet. et Neger chilensis Uromyces Diet, et Neger chilensis Valsaria Speg. chilensis Vivianella Speg. chilensis Volutellopsis Speg. chilicola Macrophoma Speg. chiliotrichi Phaeomarasmius Speg. chiloensis Psathyrella Sing. chillanensis Tubaria P. Henn. chlamydosporia Diheterospora (Godd.) Barron. et Onion (= Verticillium chlamidosporium Godd.)

chlorinella Mycena (Lange) Sing. chlorinosma Mycena Sing. chlorophanus Cortinarius Moser chloropius Rhodophyllus (Fr.) Quél. chrysanthemi Oidium Rab. chrysanthemi Phyllosticta EII, \& Dehrn.

chrysanthemi Puccinia Roze. chrysenteron Xerocomus (Bull ex St. Amans ) Quél. (Boletus) chrysogenum Penicillium Thom. chrysopellus Gymnopilus (Bres. \& Curt.) Murr. (Agaricus)

chrysosperma Cytospora Pers, ex Fr. chrysosperma Trichia DC. chrysospermum Sepedonium (Bull.) Fr.

chusqueae Aulographum Speg. chusqueae Coniosporium Speg. chusqueae Dimerosporium P. Henn. chusqueae Gloniella P. Henn. chusqueae Glonium P.Henn. chusqueae Hughesinia Lindq. et Gamund.

chusqueae Lophiosphaera Speg. chusqueae Melanomma P. Henn. chusqueae Phyllachora P. Henn. \& Lindau

chusqueae Rosellinia Speg. chusqueicola Anthostoma Speg. chusqueicola Gloniella P. Henn. chusqueicola Sphaerella Speg. chusqueophila Mycena Sing. dacrydii Mycosphaerella Butin dactylocystis Psathyrella Sing. dahliae Verticillium Kleb. dahliaecola Phyllosticta Brum. dankaliensis Gymnascella (Castell.) Currah

darvini Asterina Berk. darwini Cortinarius Speg. darwinii Cyttaria Berk. dasypus Cyathus Nees dattila Torulopsis (Kluyber) Lodder debaryanum Clastoderma Blytt. debaryanum Pythium Hesse debaryanus Mucor Schost. debilis Mycena $\mathrm{Fr}$.

decipiens Pleurage (Wint.) Kunter decipiens Phoma Mont. decipiens Trichia (Pers.) Mc Br. decosteae Phoma P. Henn. defibulatus Paxillus Sing. deflexum Myxotrichum Berkeley deformans Exoascus (Berk.) Fckl. deformans Tophrina (Berk.) Tul. degener Xerotus Fr.

delicata Clavaria $\mathrm{Fr}$.

delicata Heterochaete (Klotzsch.) Bres.

delicatum Varicosporium Iqbal deliciosus Lactarius (L. ex Fr.) S.F Gray

deliquescens Dacryomyces Fr. dematioideum Helminthosporium Bub. et Wröbl.

dematium Vermicularia (Pers.) Fr. dendritica Malbranchea Sigler \& Carmichael

dendrophora Campanella Sing. dendroseridis Uredo Jorst. emend. Linq.

dendroseridis Uromyces KeissI. 
dentriticum Fusicladium (Wallr.)

Fckl.

denudata Arcyria (L.) $\mathrm{Mc} \mathrm{Br}$.

depauperatus Saccobolus (Berk. \& Broome) Hansen

depilata Stropharia (Pers.) Fr. depressa Puccinia Diet. \& Neger depressa Tubercularia Lév. deserticola Tulostoma Phi,. desfontaineae Mycena Sing. desmazieri Ascochyta Cav. desmazieri Phoma Dur. desolationis Metasphaeria Rehm desolationis Phoma Speg. desolationis Stereum Speg. destruens Diplodia Mc Alp. deusta Sphaeria Hoffm. dianthi Colletotrichum Auct. Amer. dianthi Septoria Desm. dianthi Uromyces (Pers.) Niessl. dianthicola Alternaria Neerg. diaporthoides Skottsbergiella Petr. diatrypelloides Hypoxylon Speg. dicksoni Rhizophydium P. Weight dictyospermum Physarum List. dichondrae Aecidium Hariot dichondrae Puccinia Mont. dichrosus Cortinarius Moser dichrous Polyporus Fr. diemii Amanita Sing. difforme Diderma Pers. difforme Didymium (Pers.) Duby diffusa Laestadia (Crië) Sacc. \& Trav.

digitutum Penicillium (Fr.) Sacc. digna Puccinia Arth. \& Holw. dilabens Asterina Syd. dimorphocystis Hohenbuehelia Sing. diptychocystis Pluteus Sing. disciflorum Phragmidium (Tode) James disciformis Cyttaria Lév. discogena Mycena Sing. discolor Anthracophyllum (Mont.) Sing.

discolor Patellaria Mont. discolor Tranzschelia (Fckl.) Tranzs. \& Liv.

discophora Nectria Mont. discophora Sphaeria Mont. discors Puccinia Jacks. \& Holw. discula Helotiella Sacc. \& Trott. dispar Marasmius Mont. dispersa Puccinia Ericks. disputatum Entoloma Horak. dissectus Polyporus Lév.

echinulatum Heterosporium (Berk.) Cke.

disseminatus Coprinus (Pers, ex Fr.) echinulatus Amauroascus (Dutta \& S.F Gray (Agaricus, Psathyrella) dissimilis Rhodophyllus Sing. dissitus Polyporus Berk. \& Br. distenta Puccinia Jacks. \& Holw. ditissima Nectria Tul.

doelloi Aparaphysaria Speg. doelloi Lachnea Speg. doloris Puccinia Speg. domiciliana Galactinia (Cooke) Gamundi dothideoides Sclerotium Speg. drabae Puccinia Rudolphi drimycola Leptothyrium Speg. drimydis Actinothyrium Speg. drimydis Asterinella (Lév.) Speg. drimydis Crotone (Lév.) Theiss. \& Lyd.

drimydis Depazea Berk.

drymydis Dothidea Lév.

drimydis Lembosia Lév.

drimydis Leveillela (Lév.) Th. \& Syd. drimydis Montagnella P. Henn. drimydis Munkiella (Lév.) Speg. drimydis Phyllosticta Speg. drimydis Septoria Mont. drimytis Seynesia (Lév.) Speg. drimydis Sphaerella (Berk.) Sacc. drimydis Sphaeria Berk. drimydis Stictopatella Bat.\& Barreto drimydis Stuartella Rehm. dryopterialis Mycena Sing. dubiosa Bovista Speg. dunaliae Aecidium Lindquist dupontii Penicillium Griffin \& Maubl. duseni Fayodia (Bres.) Sing. duseni Ombrophila P. Henn. duseni Uromyces Diet. \& Neger dusenii Anthracophyllum P. Henn. dusenii Aecidium Diet. \& Neger dusenii Amphisphaeria $\mathrm{Rhm}$. dusenii Dasyscypha Rehm. dusenii Panus Bres. dusenii Rhynchosphaera P. Henn. dyctyopus Polyporus Mont. eburneus Marasmius Th. ectypus Polystictus Br. et Cav. echinatus Marasmius Rom. echinospora Galactinia (Karst.) Le Gal. echinospora Laccaria (Speg.) Sing. echinospora Peziza Karst.
Ghosh) von Arx (=Pseudoarachniotus echinulatus Dutta \& Ghosh)

echola Psilocybe (Fr.) Sing.

edulis Boletus Bull.

edulis Pholiota P. Henn.

effusa Peronospora (Grev.) Rahb. effusum Diderma (Schw.) Morgan elachus Cortinarius Moser elaeophylla Galerina Sing. elaiotus Cortinarius Moser elatum Chaetomium Kunze. \& Schm. elegans Comatricha (Racib.) Lister elongatipes Naematoloma (Peck) Sing. elzoi Melanconiopsis Speg. ellipticus Uromyces Diet. \& Neger emaculata Puccinia Schw. enceliae Puccinia Diet. et Holw. enceliae-tomentosae Uredo Maire encephala Nematelia Fr. encephaloides Amylocarpus Currey endiviae Puccinia Pass. endobioticum Synchytrium (Schilb.) Pers. endoxatha Fistulina Speg. enteroleucus Polyporus Fr. enteroxantha Diatrype Berk. entomospora Taphrina Thaxt. ephedrae Glonium P. Henn. ephedricola Mycosphaerella Butin ephedricola Pleospora Speg. epicampis Uromyces Diet. et Neger epidendrum Lycogala (L.) Fr. epilobii-tetragonii Puccinia (DC.) Wint.

epiphyllum Helotium Fr. epiphyllus Marasmius Fr. epipterygia Mycena (Scop. exFr.) S. F. Gray

episphaeria Fusarium (Tode) Syd. et Nausen $(=\boldsymbol{F}$. aquaeductuum (Tode) Snyd \& Hansen) episphaeria Nectria (Tode) Fr. epixanthum Hypholoma Fr. epixyla Vermicularia Fr. equinum Trichophyton Gedoelst. equinus Lasiobolus (Gmel.) Karst. equorum Coprolepa Fckl. erebia Pholiota Fr.

erebius Cortinarius Moser. 
eringiicola Sphaerella Speg. eryngiicola Paranthostomella Speg. erysiphoides Oidium Fr.

erythrellus Leucoagaricus (Speg.) Sing.

erythropus Marasmius Fr. sensu Mont. escalloniae Phaeophacidium P. Henn. et Lindau

escalloniae Phyllachora Pat. escalloniae Trabutia (P. Henn.) Theiss.

esculenta Morchella (L.) Pers. espinosae Aecidium Syd. espinosae Caeoma Syd. espinosae Clitocybe Sing. espinosae Cyttaria Lloyd. etchellssii Torulopsis Lodder. et Kreger. Van Rij. eucryphiae Pluteus Sing. eucryphiarum Mycena Sing. eugeniicola Didymosphaeria Speg. euopia Puccinia Syd. euphorbiae Melampsora (Schum.) Cast.

euphorbiae Uromyces Cke. \& Peck. eustoma Leptosphaeria (Fr.) Sacc. evolceanui Chrysosporium (Rhandhawa. et Sandhu) Carmich. evonymi-japonici Oidium (Arcang.) Sacc. excavata Sphaeria Fr. excruciatus Cortinarius Moser exiguus Marasmius Sing. exilis Meliola Syd. eximia Patellaria Syd. exitiosus Polydesmus Kühn. exotica Septoria Speg. expansum Penicillium (Lk.)Thom. exquisita Naucoria Berk. (Agaricus) fabae Uromyces (Pers.) D'By. fabea Botrytis Sard. fagaciarum Ceratocystis (Bretz.) Hun fagi Mikronegeria Diet. \& Neger fagi Melampsora Diet. \& Neger fagi Scolecosporium Lib. fagicola Septoria Speg. faginea Propolis (Schrad.) Karst. fallax Odontia (Fr.) Oud. famata Torulopsis (Harrison)Lodder. \& Kreger Van Rij.

furinaceum Didymium Schrad. farinaceum Hydnum Pers. farinaceum Physarum Pers. farinosa Isuria (Dicks.) Fr. farinosum Oidium Cke.

Corner.

fasciculare Hypholoma Huds. (Agari flavocomum Physarum Berk. cus)

favoginea Trichia (Batsch) Pers. fennica Clavaria Karst. flavolanata Nectria Berk. et $\mathrm{Br}$. flavum Oncocladium Wallroth flavus Aspergillus Link ex Gray flavus-orizue Aspergillus Link. flectens Uromyces Lagh. van Oorschot fernandeziana Amanita Sing. fernandeziana Limacinia Neger. fernandeziana Mycena Sing. fernandezianus Coriolus Sing. (Polyporus, Polystictus)

ferruginea Hymenochaete (Bull.) Mass.

ferruginea Cercospora Fuckel ferruginea Stemonites Ehr. ferruginosa Poria (Schrad.) Fr. ferruginosa Tubifera (Batsch) J.F. Gmelin.

festucae Puccinia (DC.) Plowr. fibriatum Dematium Schw. flexuosa Licea Pers.

flocculenta Cytidia (Fr.) Hönh. \& Listch.

floculosipes Stropharia Sing. floriforme Geastrum Vitt. floriformis Trichia (Schw.) G.Lister floccipes Hydropus (F.) Sing flotoviae Leptosphaeria Speg. flourensiae Marsonia Speg. flourensicola Septoria Speg. flourensiicola Phyllosticta Speg. fluorescens Ascophanus Speg. fluorescens Cortinurius Horak foeda Sphaeria Lév.

fibula Gerronema (Agaricus, Ompha- foeniculina Diplodina Speg. lia) (Bull. ex Fr.) Sing. foeniculina Sphaerella Speg. fibuliger Endomycopsis (Lind.) Dekk. foenisecii Paneolina (Pers. ex Fr.) fici Cerotelium (Cast.) Arth. Maire filiformis Anguillospora Greathead filiicola Dimorphospora Tubaki filum Calocera Lèv

filum Clavaria Lév. filum Darluca (Biv.) Cast fimbriata Ceratocystis f. platani Walter

fimetarius Coprinus $\mathrm{Fr}$. foetida Tilletia (Wallr.) Liro foliicolum Hysterium Fr. fomentarius Boletus $\mathrm{L}$. fomentarius Polyporus Fr. fornicatum Geastrum (Huds, ex Pers.)Hook.(Geaster) fragariae Mycosphaerella (Tul,) Lind.

fimiseda Pleurage (Ces. \& de Not.) fragariae Oidlium Harz. Griff.

fitzroyae Appendiculella Butin fitzroyae Chloroscypha Butin flaccida Lenzites (Bull.) Fr. flaccida Ramaria (Fr.) Ricken. flacida Puccinia Bert. \& Br. flummea Exidia Berk.

fragariae Phytophthora Hickman. fragariae Sphaerella (Tul.) Sacc.

fragariae Ramularia Peck. fragariae Septoria Desm. fragariae Sphaeria Fckl. fragiformis Sphaeria Pers. fragile Myscenastrum Lév. fragile Thaxterogaster (Zeller. \& Dodge) Smith apud. Sing. flammipes Pluteus Horak flummuliformis Psilocybe Sing. fragile Scleroderma (Lév.) Sacc. flammuloides Cortinarius Horak. \& fragilis Leocarpus (Dicks.) Rost. Moser

flareri Candida (Cif. \& Rect.) Lang. \& Guerra

fragilis Hymenogaster Zeller. \& Dodge

fragilis Pila (Lév) Speg. fragilis Saccharomyces Jörg.

flava Clavaria Schaeff.
flava Malbranchea Sigler \& Carmich. flava Ramaria (Fr.) Quél.

francoae Leptosphaerella Speg. francoae Leptosphaeria Speg.

flavidum Scleroderma Ell. \& Everh. francoae Phomopsis Speg.

flavipes Aspergillus Bainer \& Sartori frecuentans Penicillium Westling flavobrunescens Ramaria (Atk.)

$$
\text { (=P. glabrum (Wehmer) }
$$


Westling freindlingiae Pholiota (Sing.) Sing. freticola Aposphaeria Speg. freticola Pleospora Speg. freticola Pyrenophora Speg. friesii Trematosphaeria Rehm. frondosus Polyporus Fr. frowardii Pholiota (Speg.) Horak fructiculosa Ceratiomyxa (Müll.) Mc Bridge

fuciformis Tremella Berk. fuckeliana Sclerotinia (Di By.) Fckl. fuchsiicola Phyllosticta Speg. fuegiana Clavaria Speg. fuegiana Collybia Sing. fuegiana Diaporthe Speg. fuegiana Endogone Speg. fuegiana Gautieria Horak fuegiana Harknessis Speg, fuegiana Pistillaria (Speg.) Corner fuegiana Pleosphaeria Speg. fuegiana Pleospora Speg. fuegiana Trichospora Speg. fuegiana Xylaria Speg. fuegianum Anthostoma Speg. fuegianum Coniothyrium Speg. fuegianum Cylindrium Speg. fuegianum Cytosporium Speg. fuegianum Geomonium Speg. fuegianum Helotium Speg. fuegianum Hysterographium Speg. fuegianum Leptothyrium Speg. fuegianum Lophiostoma Speg. fuegianus Polyporus Speg. fuegianus Uromyces Speg. fuliginea Exidia Mont. fuliginea Sphaerotheca (Schlecht.) Salm.

fuligineovenosus Pluteus Horak fulva Nannizzia Stockdale fulvescens Aphanoascus (Cooke) Apinis

fulvicolor Polystictus Speg. fulvum Cladosporium Cooke. (=Fulvia fulva (Cooke) Ciferri) fumaginea Torulopsis Speg. fumago Cladosporium Link. fumago Napicladium Speg. fumigatus Aspergillus Fres. fumosa Laboulbenia Thaxt. fumosus Polyporus (Pers.) Fr. funariophilum Pachyolepyrium (Moser) Sing. funebris Mycenella Sing. funerea pestalotia Desm.

funiculosum Penicillium Thom. furfuracea Tubaria (Pers. ex Fr.) Gillet

furfuraceus Ascobulus Pers. ex Hook. furfurosum Lentinus Fr. furtiva Anguillospora Descals fusca Stemonitis Roth.

fusco-badius Polyporus Bres. fuscomarginatus Phaeomarasmius Sing.

fuscum Hypoxylon Per. ex Fr.

fusipes Tricholoma Horak gaduae Balansiopsis (Rehm) Kühn. galegae Uromyces (Opiz) Sacc. galericulata Mycena Scop. galerinoides Laccaria Sing. galii Pactilia Allesch. et P. Henn. galii Puccinia Schw.

galii Uredo Rab.

galiorum Puccinia Lk.

galligena Nectria Bres.

galligera Leptosphaeria Keissl.

gallinae Achorium Sbour. ( = Microssporum gallinae (Megn) Grig.) gamocarpae Puccinia P. Henn. gardoquiae Asterella Syd. gardoquiae Puccinia Diet. \& Neger gargal Grifola Sing. gavana Crinula Mont. gayana Cyphella Lév. (Agaricus, Flagelloscypha)

gayanus Cyathus Tul. gayanus Polyporus Lév. gayii Melanotus (Pilat) Sing. geanthi Aecidium Diet. et Neger gelatinosa Hypocrea (Tode) Fr. gemellipara Gyoerffyella Marvanova gemmata Amanita (Fr.) Gillet. gemmatum Lycoperdon Batsch genuina Phaeodropezia Le Gal geoderma Poria Speg. geosmus Cortinarius Moser geranii Puccinia Lév. geranii-silvatici Puccinia Karts. geraniicola Uromyces Speg. gerronematoides Hydropus Sing. giganteum Crocicreas Mont. giganteum Trichosporum Unna giganteus Hybogaster Sing. giganteus Polyporus (Pers.) Fr. gigas Podospora Lind. giliae Sphaerulina Speg. gilliesi Gloniella Speg. gilliesi Phaeosperma Speg. gilliesiana Diaporthe Speg. glabella Lepista (Speg.) Sing. glabrata Torulopsis (Anderson)Lodd. \& De Vries. ( = Candida glabrata (Anderson) Meyer \& Yarrow glabripes Hydropus Sing. gladioli Septoria Pass. gladiolorum Botrytis Timm. glandulosa Exidia (Bul.) Fr. glauca Botrytis Spreng. glaucum Coremium glaucum Penicillium Link. glaucum Pseudohelotium Speg. glaucus Aspergillus Link. (¿Grupo?) (= Eurotium herbariorum (Wigger) Link glaucus Pluteus Sing. gliocyclum Thaxterogaster Horak. glioderma Limacella (Fr.) R. Maire. globifera Pulvinula (Berk. \& Curt.) Le Gal. globiger Pluteus Sing. \& Digilio globosa Torulopsis (Olson. \& Hammer) Lodder. \& Kreger Van Rij. (=Candida globosaYarr.\&Mey.) globuliferum Physarum (Bull.) Pers. gloeosporioides Colletotrichum Penz. glomerula Mucor (Bainer) Lendner glossoides Dacryomitra glumarum Dicaeoma (Erikss. \& P. Henn.) Arth. \& Fromme glumarum Puccinia (Schm.) Erikss. \& P. Henn.

glutinis Rhodotorula (Fres.) Harrison gnaphalii Puccinia (Speg.) P. Henn. gnaphalii Uredo Speg. gomez-millasii Hygrocybe Lazo gossypii Oidiopsis (Wakef.) Raych. gossypii Ovulariopsis Wakef. gossypinulus Agaricus Speg. gossypinulus Marasmiellus (Speg.) Sing. gracile Heterosporium (Wallr.) Sacc. gracilis Clathrus (Berk.) Schlecht.

(Ileodyction)

gracilipes Cortinarius Moser gracilis Xylaria Keissl. gracillima Omphalia Weinm. graminea Volucrispora graminearum Fusarium Schwabe. graminella Puccinia (Speg.) Diet. \& Holw.

graminum Fusarium Corda. 
gramineum Helminthosporium Rab. graminis Erysiphe DC. graminis Ophiobolus Sacc. graminis Phyllachora (Pers.) Fckl. graminis Puccinia Pers. graminis Sphaeria Pers. graminis Scolecotrichum Fckl. graminocola Pterula (Bourd. \& Galzin) Sing.

grammica Xylaria Mont. grandjotii Uromyces Graum. graminum Dinemasporium Lév. ( $=$ D. strigosum (Pers.ex Fr.) Sacc.)

granulata Coprobia (Bull. ex Fr.) Boud.

granulata Humaria (Bull.) Quél. granulatus Suillus (Boletus) (L. ex Fr.) O. Kuntze.

granulosa Campoa (KI.) V. Arx. granulosa Dothidea Klotzsch. granulosus Marasmius Rom. grassa Isaria Pers.

gravida Culicidospora Petersen gregaria Dothiorella Sacc. gregarium Helotium Boud. gregoriana Tubaria (Speg.)Sacc. gregorianus Agaricus Speg. grisea Humicola Traaen grisea Madurella McKinnon, Ferrada. \& Montemayor. grisea Omphalia Fr. griseomellea Armillariella Sing. griseopallida Omphalina (Gesn.) Quél.

grossa Clavaria Pers. grossulariae Aecidium Schum. grylli Entomophthora Fres. guaitecaensis Polyporus P. Henn. guanaci Humaria Rehm guevinae Gloeosporium Speg. guevinae Macrophoma Speg. guevinae Phoma Speg. guevinicola Phyllosticta Speg. guillermondii Pichia Wickerham (= anamorfoCandida guilliermondii (Cast.) Lang. \& Guerra gunnerae Ovularia Speg. gunnerae Phyllosticta Speg. gunnerae Sphaerella Speg. gunnerae Ustilago Clint. gypsea Nannizzia (Nann.) Stockdale (=Arthroderma gypseum Weitz. et al.) gypseum Microsporum (Bodin) Guiart \& Grigorakis

haastii Entoloma Stevenson

habranthi Puccinia Diet. \& Neger

haematopus Mycena (Fr.) Quél.

halophilum Aecidium Speg.

halstedii Plasmopara (Farl.) Berl. \& de Toni

halstedii Peronospora Farl.

hansenii Debaryomyces (Zopf)

Lodder. \& Kreger. Van Rij.

haplopappi Diplodia Allesch.

hariotana Phoma Wint.

hariotana Sphaerella Speg.

harioti Calycela Boud.

hariotii Cyttaria Fish.

hartigii Pestalotia Tubeuf

helatioides Guepinia Berk.

helianthi Puccinia Schw.

helminthobasis Mycena Sing.

helvola Clavaria Pers.

hemimycena Marasmius Sing.

hemisphaerica Urophlyctis (Speg.) Sing.

hemisphaerica Uromyces (Speg.) Syd.

hendersonii Coprinus Berk.

hepaticus Cerionyces Sacc.

heptamera Sporormia Awers.

heraclei Erysiphe DC. ex Saint-Aman herbariorum Eurotium (Wigg.) Link. herbarum Helotium (Pers.) Fr. herbarum Peziza Pers.

herbarum Pleospora (Pers.) Rab.

herbarum Torula Link.

herquei Penicillium Bainier\& Sartori herbarum Cladosporium (Pers.) Lk. hesperidum Phoma Mc Alp.

heterocystis Galerina (Atk.) Smith. \& Sing.

heterochroma Cortinarius Moser heteromorpha Puccinia Speg. heterospora Cryptosphaerina Speg. heterosporium Fusarium Nees. heterosporum Gliostroma Mont. heterosporum Hypoderma Butin. heterosporum Mucor Fischer hidropiperis Sphacelotheca (Schum.) D'By.

hiemalis Coccomyces Higg. hiemalis Mycena (Obs. in Retz. ex Fr.) Quél.

hieraciatum Dicaeoma (Jacks.) Arth. hieracii Puccinia (Oud.) Jorst. hierochloae Hendersonulina Speg. hieronymii Ravenelia Speg. hippeastri Aecidium Gaum. hirsutulus Polystictus Schw. hirsutum Stereum (Willd ex Fr.) S. F. Gray (Thelephora)

hirsutum Trichoglossum (Pers. ex Fr.) Baud.

hirsutus Polyporus Fr.

holci Tilletia (West) De Toni holcina Puccinia Erikss.

holmii Torulopsis (Jörg.) Lodder (= Candida holmii (Jörgensen)

Meyer \& Yarrow)

hololeucum Entoloma (Sing.) Horak homoianthi Puccinia Sid.

honoratoi Rhizopogon Speg. hookeri Anthracoderma Speg. hookeri Coniothyrium Speg. hookeri Cortinarius Speg. hookerii Cyttaria Berk. hordei Ovularia (Cav.) Sprague hordei Puccinia Ottk. hordei Ustilago (Pers.) Kell. \& Sw. hornitophila Hypocopra Speg. hortensiae Oidium Jorst. hosaticus Sporobolomyces Windisrh. hualtatinum Aecidium Speg. humarioides Cheilymenia (Rehm.) Gamundi

humarioides Lachnea Rehm humboldtiana Melampsora Speg. humuli Pseudoperonospora (Miy. \& Tak.) G. W. Wils.

humuli Sphaerotheca (DC). Burr. hyalinotricha Mycena Sing. hyctemerus Coprinus Fr. hydnoides Ceratium (Jacq.) Alb. \& Schw.

hydrocotyles Puccinia (Lk.) Cke. hydrocotyles Uredo Mont. hydrocotylinum Aecidium Speg. hydropiperis Ustilago (Schum.) Schroet. hygrocyboides $C_{y p t o t r a m a}$ Sing. hygrometricum Geastrum Pers. hygrophilus Mucor Ond.

hymenoglium Stereum Speg. hyoscyami Peronospora D' By. hyperici-frondosi Uromyces (Schw) Arth. hypericinum Diploceras Diet. hyperopta Stemonites Meylan. hypertropha Helechora Sherwood 
hypnophilus Pleurotellus (Pers. ex

Berk.) Fayod

hypnorum Galera Batsch

hypochoeridis Puccinia Oud.

hypomiltum Hypoxylon Mont.

hypomilla Sphaeria Lév.

hypoxantha Dermocybe Horak

hypoxylon Sphaeria Pers.

hypoxylon Xylaria (L.) Grev,

hypsophilum Aecidium Speg.

hysterinum Tryblidium Duf.

hysterioides Lophodermopsis Speg.

hysterioides Lophodermium (Pers.)

Sacc.

idaei Physalospora Sacc.

idiolens Mycena Lundell

igniarius Boletus L.

igniarius Polyporus Fr.

illaudanta Uredo Jacks. \& Holw.

illitus Cortinarius Moser \& Horak

imberbis Peziza Bull.

imbricatum Tricholoma Fr.

immersus Ascobulus Persoon ex Fr. impedidum Entoloma Horak

imperfecta Ascochyta $\mathrm{Pk}$ (= Phoma medicaginis Malbr. \& Roum.)

impersicum Acanthostigma Speg.

impudicus Phallus L.

inaequalis Venturia (Cke.) Wint.

inaequialtus Uromyces Lasch

incarnata Arcyodes (Alb. \& Schw.)

O.F. Look.

incarnata ArcyriaPers.

incarnatus Lachnobolus (Alb. \& Schw.) Schroet.

inconspicua Flammula Bomm. \& Rouss.

inconspicua Torulopsis Looder \& Kreger Van Rij (Candida)

incrustatus Clitopilus Sing. in Sing. \& Digilio

indicum Chrysosporium (Randhawa \& Sandhu) Garg

inermis Polyporus Ell.

inermis Poria Ell. et Everh.

infectoria Pleospora Fckl.

infestans Phytophthora (Mont.) D'By.

infirmum Entoloma Horak

inflata Geniculospora (Ingold) Nils. ex Marv. \& Nils

inflatipes Cortinarius Moser

inflexus Marasmius Mont.

inocybiforme Tricholoma Horak inquinans Bulgaria (Pers.) Fr. inquinans Coniosporium Dur. \& Mont.

insignis Arcyria Kalchb. \& Cke. insignis Mycena A.H Snith insignis Sordaria E.C.Hans (=Strattonia insignis (E.C.Hans.)Lundq. intermedia Aschersonia Petch. intermedia Candida (Cif. \& Ashf.) Lang. \& Guerra

intermedia Cyttaria Palm.

intermedia Pleospora Speg.

intermedia Sporormia Auerswald (Sporormiella)

intermedium Pythium D'By. intermedius Gymnoascus Orr interveniens Puccinia (Pk.) Bethel invisa Uredo Speg.

involuta Psathyrella (Romagn.) Moser in Gams

involutus Paxillus Batsch iridis Didymium (Ditm.) Fr. italicumPenicilliumWehm jacobsthalii-henriciAecidium Magn. jaffueli Diaporthe Speg.

jaffueli Gloniella Speg.

jaffueli Metasphaeria Speg.

juffueli Phyllosticta Speg.

jaffueli Pluteus ( Speg. )Sing . (Mycena, Naucoria) jaffueli Sphaerella Speg. jaffueli Septoria Speg. jaffueli Trochila Speg. jaffueli Tuberculina Speg. jaffueli Tubaria (Speg.) Sing. jaffueliana Eutypella Speg. jaffucliana Puccinia Speg. jaffuelianum Calothyriolum Speg. jafueli Didymella Speg.

johovii Cyttaria Espinosa japonicum Exobasidium Shir,

jensenii Ustilago Rost.

johowii Uromyces Diet. \& Neger

josserandii Dermoloma Dennis. \& Horton

juanensis Conocybe (Henn.) Sing.

juaniicola Mycena Sing. jubaeae Aposphaeria Speg. jubaeae Leptosphaeria Speg. jubaeae Leptostroma Speg. juglandicola Phoma Speg. juglandicola Valsa Speg. juglandina Hendersonia Speg. juglandis Mursonia (Lib.) Sacc. juncacearum Leptostroma Sacc.

junci Uredo Strauss

junci Uromyces (Desm.)Tul.

juncina Dothichiza Speg.

juniperinus Marasmiellus Murr.

jurei Geastrum Lazo

jussiaeae Septoria Ell. \& Kellerm.

kalchbrenneri Septoria Sacc.

keissleri Uredo Lindq.

keratinophilus Aphanoascus Punsola \& Cano

keratinophilus Chrysosporium D. Frey ex Carmichael

kerguelensis Peziza Berk.

kerguelensis Scutellinia (Berk.) Le Gal

kloeckeri Debaryomyces (Gill.) Péju

koeleriae Puccinia Arth.

krusei Candida (Cast.) Basgal

kuhneriana Collybia Sing.

kuhneriana Conocybe Sing.

labecula Asterina Mont.

labens Mycrocyclus

laccata Laccaria (Scop. ex Fr.) Berk. $\& \mathrm{Br}$.

lacera Cyphella Fr.

lacrymans Merulius (Jacq.) Fr.

lactea Thelephora $\mathrm{Fr}$.

lactea Russula (Pers.) Fr.

lacteum Corticium Fr.

lacteus Irpex $\mathrm{Fr}$.

lacteus Leptomitus Agardh.

lactis Oospora (Fres.) Sacc.

(= G. candidum Link)

lactis-condensi Torulopsis (Hammer) Lodder. \& Kreger Van Rij.

lactucae Bremia Bregel

laeensis Isthmotricladia Matsush.

lactifolius Cortinarius Horak

laevis Ustilago Kell. \& Swing

laevis Tilletia Kühn

laevissima Psathyrella (Romang.) Sing.

lageniformis Sporormia Fckl.

(Sporormiella)

langei Lyophyllum Sing.

langloisii Physalacria Ell. et Everh.

lamuginosa Humicola (Griff. \& Maubl.) Bunce

lamuginosa Niesslia Butin

lamuginosus Thermomyces Tsiklinsky

lapageriae Pestalotia P. Henn.

lapageriae Phoma P. Henn.

lapageriae Physalospora Speg. 
lapageriae Pleospora Speg. lapageriae Sphaerella Speg. lapageriicola Cryptostictis Speg. lapsanae Puccinia (Cke.) Fckl. lardizabalae Phoma Speg. lardizabalae Physalospora Speg. lardizabalae Sphaerella Speg. lardizabalicola Phoma Speg. larici-populina Melampsora Kieb. lateralis Paxillus (Schaeff.) Sacc. lateritia Trichia Lév. lateritiorosella Hygrocybe Sing. lateritium Physarum (Berk. et Rav.) Morg.

lateritium Verticillium Berk. lateritium Xerotus Berk. et Curt. lathyrinum Aecidium Seg. lathyrinum Polythrincium lathyrinus Uromyces Speg. laetifolius Cortinarius Horak. laureliae Melanops Syd. laurentii Cryptococus (Kuff.) Skinner laxa Monilinia (Aderh. \& Ruhl.) Honey.

laxa Monilia (Ehr.) Sacc. \& Vogl. laxa Sclerotinia Aderh. \& Rud. lazoi Agrocybe Sing. lazoi Arachnion Demoulin lazoi Cortinarius Moser lazoi Entoloma Horak lazoi Mycena Sing. lazoi Psilocybe Sing. lazulinus Cortinarius Horak \& Moser lazulinus Rhodophyllus (Fr.) Quél. leathsi Laboulbenia Speg. lecideola Patellaria Fr. lenticulare Helotium Bull. leprosa Fairmaniella(Fairm.) Petr. \& Syd.

leptaula Trametes Speg. leptocystis Cortinarius Moser leptockloaePuccinia Arth.\&Fromme leptodon Hydnum Mont. leptosperma Sphaerella Speg. leptosperma Vermicularia Speg. leptospora Phoma Speg. leptosporum Phaeosperma Speg. leuceriicola Diaporthe Speg. leuceriicola Puccinia Syd. leucobucha Peziza Alb. et Schw. leucoconium Oidium Desm. leucoloma Humarina (Hedw. ex Fr.) Seaver leucomela Peziza Pers. leucomelaema Helvella (Pers.) Nannf.

leucophaea Trichopeziza Rehm. leucophaeus Fomes Mont. leucophaeus Physarum Fr. leucopus Helotium Mont. leucostoma Cytospora (Pers.) Sacc. leucostoma Valsa Pers.

leucostylum Helminthosporium Drechs. ( = Bipoluris nodulosa (Berk \& M.A.Curtis) Shoem.)

leucotricha Podosphaera (Ell. \&

$$
\text { Everh.) Salm. }
$$

leve Corticium Pers.

leveilleanum Aecidium Magn.

leveillei Puccinia Mont.

liberta Puccinia Kern.

libertiae Asterina Syd.

libertiana Sclerotinia Fckl.

lidenuthianum Colletotrichum (Sacc. \& Magn.) Br. \& Cav.

lignaria Coniochaetia (Grév.) Masse. lignifragum Coniosporium Mont. lignyota Karschia (Fr.) Sacc. lignyotus Cortinarius Horak lilacina Calvatia (Berk. \& Mont.) Lloyd.

lilacina Bovista Mont. lilacipes Entoloma Horak limbatus Polyporus Fr.

limonea Armillariella Stev. limonii Uromyces (DC.) Lév.

limonioides Cortinarius Moser

limulatellus Phaeomarasmius Sing. lindemuthianum Colletotrichum (Sacc. \& Magn.) Br. \& Cav. lindemuthianum Gloeosporium Sacc. \& Magn.

linearis Uredo Pers.

lingue Anthostomella Speg. lingue Cercospora Speg. lingue Laestadia Speg. lingue Leptosphaerella Speg. lini Fusarium Bolley (=F.oxysporum Schlecht.)

lini Melampsora (Pers.) Lév. linkii Rhodophyllus (Fr.) Quél. lintearia Sphaeria Mont. lithraeae Botryodiplodia Speg. lithraeae Diaporthe Speg. lithraeae Sphaerella Speg. lithraeae Septoria Speg. litorale Microthyrium Speg. litorale Myocopron Speg. littoralis Gymmnascella (Orr) Currah lividum Helotium Mont.

loasae Septoria Speg.

lobata Puccinia Diet.

lobeliae Heterosporium Speg.

locaniensis Lepiota Espinosa.

loculata Diplodia Mont.

Iomatiae Phoma Speg.

longibrachiata Clavatospora (Ingold)

Nils ex Marv.\& Nils.

longinqua Galerina Smith. et Sing.

longinquus Panellus (Berk.) Sing.

(Agaricus, Pleurotus)

longispora Zignoella Speg.

longissima Anguillospora (Sacc. \& Sud.) Ingold

lophiostomoides Anthostoma Speg.

lorentziana Dothidea Speg.

lotaensis Marasmius P. Henn.

loyita Boletus Horak

loyo Boletus Phil. ex Speg.

lugdunensis Heliscus Sacc. \& Thierry

lugubris Propolis Speg.

Iumae Leptosacca Syd.

lumae Mycosphaerella Syd.

lumae Phomopsis Syd.

Iupulina Uredo Speg.

Iutea Myceliophthora Cost.

luteo-badium Stereum Fr.

luteo-cincta Coniophora (Berk.) Cke. luteofuscum Callistosporium Sing.

luteola Sicalis

luteolus Rhizopogon Fr.

lutescens Tremella Pers.

luteus Suillus (L.ex Fr.) S. F.Gray. (Boletus)

Iuxurians Puccinia Diet. et Neger

lyallii Diderma (Mass.) Mc. Br.

lycii Puccinia Bert.

lycoperdon Reticularia Bull.

lycopersici Fusarium Sacc.

ycopersicum Oidium Thuem.

lythri Aecidium Diet. \& Neger

macella Uredo Jacks. \& Holw.

macilentus Cortinarius Moser

macquariensis Galerina Smith. \& Sing.

macrachaenii Puccinia Diet.\& Neger macrocarpa Badhamia (Ces.) Rostaf. macrocarpa Cribraria Schrad. macrocarpa Nidula Lloyd. macrocarpum Cladosporium Preus. macropoda Septoria Pass. macrospora Disciseda Speg. 
macrospora Gelopellis Zeller macrospora Metasphaeria Speg. macrospora Niptera Boud. macrospora Puccinia Diet. macrosporum Aecidium Diet. et Neger macrosporus Kuehneromyces Sing. macularis Asterella Syd. macularis Septoria Lév. maculatissimus Polyporus Lloyd. maculatum Entomosporium Lév. maculiforme Circinotrichum Nees. madiae Coleosporium Cke. madiae Puccinia Cyd. mafilensis Microdiplodia Speg. magellanica Bovista Speg. magellanica Cercospora Speg. magellanica Diaporthe Speg. magellanica Gnomonia Speg. magellanica Milesina (Fall.) Hratsuka magellanica Milesia Faull. magellanica Phyllosticta Speg magellanica Physalospora Speg. magellanica Placosphaeria Speg. magellanica Pleospora Speg. magellanica Puccinia (Berk.) Speg. magellanica Rosellinia Speg. magellanica Sphaerella Speg. magellanica Uncinula Thaxit. magellanicum Aecidium Berk. magellanicum Cystothyrium Speg. magellanicum Fusidium Speg. magellanicum Hypoxylon Speg. magellanicum Hysterographium Speg.

magellanicum Lophiotrema Speg. magellanicum Microsporum Caretta

\& Piontelli

magellanicum Thaxterogaster Sing. magellanicum Tricholoma Speg. magellanicus Agaricus Speg. magellanicus Cortinarius Speg. magellanicus Uromyces Spcg. majalis Pholiota Sing. major Urophlyctis Schroet. majuscula Pleuroflammula Sing. majusculum Corticium Spcg. malachrae Erysiphe Seaver. mali Phomopsis Robert. malicroticis Neofabraea (Cordley) Jack

malorum Physalospora Shear malorum Sphaeropsis Peck. malvacearum Puccinia Bert. exMont. mammaeformis Sphaeria Pers. manivana Mycosphaerella Butin maqui Septoria P. Henn.

marconi Dendrophoma Cav. marchalianum Tetracladium de Wild. marchii Hygrocybe (Bres.) Sing. margaritiferum Trichosporum (Stautz) Buchw. margaritisporus Cortinarius Moser marginata Dothidea Lév. marginata Pholiota Batsch. marginatum Hypoxylon (Schw.) Berk.

mariluanensis Marasmiellus (Speg,) Sing. mariluanensis Mycena Speg. mariluanensis Naucoria Speg. maritima Renispora Linder maritimum Zalerion (Linder.) Anastasiou

maritzburgensis Hysterostoma Bat. \& Vital

marmoratus Polyporus Berk. marthae Cystolepiota Sing. marthae Galerina Sing. marthae Pholiota Sing. marthae Psilocybe Sing. martii Erysiphe Lév. martii Fusarium Appel. \& Wr. martii-phaseoli Fusarium Berk. martipanis Pseudoclitocybe Sing. masatierrae Uredo Lindq. matildae Pestalotia Mujica maulensis Cortinarius Moser maydis Puccinia Bereng. maydis Sirocuccus Speg. maydis Ustilugo Cda. mayteni Montagnella P. Henn. mayteni Phyllosticta Speg. mayteni Septoria Wint. media Pleospora Miessl. medicaginis Cercospora Ell. \& Ev. medicaginis Pseudopeziza (Lib) Sacc. mediofuscum Agaricus (Moller) Moller medulla-panis Poria (Pers.) Fr. medusa Lulworthia (Ell \& Everh.) Cribb.

medusae Melampsora Thuemen megalosperma Pholiota Sing. megalospermus Uromyces Speg. megalospora Rutstroemia (Speg.) Gamundí

megalospora Sporormia Awers. megalosporum Helotium Speg. megalosporum Miriopetalum Sing. melaleucum Tricholoma Speg. melaleucus Phellodon (Fr.) Karst. melaloma Anthracobia (Peziza)

(Alb. \& Schw. ex Fr.) Boud melandryi Uromyces Diet. \& Neger melanogramma Schizonella (DC.) Schroet.

melanosarx Melanoleuca Sing. melanospermum Didymium (Pers.) $\mathrm{Mc} \mathrm{Br}$.

meliloti Stagonospora (Lasch.) Petr. melinii Candida Diddens. \& Lodder melinoides Agaricus Fr.

melonis Mycosphaerella (Pass.) Chin. \& Wlker. mellea Clitocybula Sing. mellea Armillaria (Vahl.) Quél. mellea Puccinia Diet. \& Neger mellea Tremella

melleomitis Cortinarius Moser et Horak

membranaceum Hydnum Bull. mentagrophytes Trichophyton (Robin) Gedoelst.

menthae Puccinia Pers. menziesi Camarosporulum Speg. merdaria Psilocybe (Fr.) Ricken. merdarium Chrysosporium (Link ex Grev.) Carmichael merulius Agaricus Bert. mesenterica Tremella Retz. mesophaeum Hebeloma (Pers.) Quél. mesophaeus Cortinarius Moser mesospermum Entoloma Horak metallica Calomyxa (Berk.)

Nicuwland metallica Margarita (Berk. \& Br.) List. metuloidiphera Mycena Sing. mexicana Hygrocybe Sing. meyeri-alberti Puccinia P. Magn. micaceus Coprinus (Bull) Fr. microalba Mycena Sing. microaquatica Dactyella Tubaki microcarpa Pholiota Sing. microcephtala Xylaria Mont. microleuca Mycena Sing. microleucurum Coniosporium Speg. micromamma Mycena Speg. microscopica Asterina Lév. microscopicum Microthyrium Desm. microsora Cercospora Sacc. microspora Cytospora (Cda.) Rabh. 
microspora Neoclitocybe Sing.

microspora Phoma Pat.

microsporium Glonium Sacc.

microstomus Ophiobolus Speg.

microthelia Puccinia Syd.

micrura Sordaria Speg.

miehei Rhizomucor (Cooney \&

Emers.) Schipper

miersi Monochaetia Speg.

miersi Macrophoma Speg.

miersiae Uromyces Gäum.

millae Aecidium Jacks \& Holw.

millei Ductifera Lloyd.

miniatum Lycogala Pers.

minima Mycenella Sing.

minima Sporormia Awd.

minimum Trichocladium De Hoog. \& Grinbergs

minimus Geastrum Schw (Geaster)

minimus Saccobolus Vel.

minitum Gerronema (Sing. \& Digilio) Sing.

minor Naemacyclus Butin (= Cycloneusma minus (Butin) Di Cosmo, Peredo \& Minter)

minor Sclerotinia Jagger

minor Uromyces Schroet.

minus Didymium (A. Lister) Morgan

minuta Rhodotorula (Saito) Harrison

minutissima Gerronema Sing.

minutula Psathyrella

minutulia Mycena Sing.

mirabile Dictydium (Rost.) Meylan

miser Coprinus Karst. sensu Langc

mitis Pleurotus Pers.

mitrariae Asterina Syd.

modesta Lactocollybia Sing.

modestus Polyporus Kunze

moelleri Galerina Bas.

moelleriana Rumaria (Bres.\& Roum.) Corner

molischiana Torulopsis (Zikes)

Lodder

mollis Crepidotus (Fr.) Staudc

molluscus Boletus Pcrs.

molluscus Polyporus Pers.

moniliforme Ceratocystis (Hedge)

Moreau

moniliformis Fusarium Sheld.

monilioides Oidium (Need) Lk.

monocarpa Ceutospora Mont.

monochactoiles Coryneum Speg.

monophyllum Dematium

monosporus Triscelophorus montagnei Armillariella Sing.

montana Pholiota Sing.

montana Psilocybe (Pers. ex Fr.)

Kummer

montanensis Puccinia Ell.

montivaga Galerina Sing.

morchellaeformis Gymnopaxillus Horak

morchellocephalus Merulius Bert.

morchellocephalus Stylobates

moriformis Sphaeria Lév.

morthieri Massarina (Fuck.) Arx \& Mueller

mucedo Mucor (L.)Fres.

mucida Isaria Pers.

mucilaginosa Rhodotorula (Jörg.)

Harrison

mucilago Spumaria Pers,

mucor Agaricus Batsch

mucronatum Phragmidium (Pers.)

Schlecht

muehlenbeckiae Aecilium Jacks \&

Holw.

mulinii Uromyces Schroct.

multiceps Sphaeria Sow.

multiplex Xylaria Kuntze

multiseptata Gloniella Speg.

mundula Rhodocybe (Lasch) Sing.

mundulum Aecidium Jacks. \& Hlw:

munyozii Marusmius Sing.

muscae Empusa (Fr.) Cohn

muscae Entomophthora (Cohn)Fres.

muscicola Phy'sarum

muscigena Anthina Speg.

muscorum Peziza Holmsk.

mutila Diplodia Fr.

mutisiae Phyllachora Speg.

myceliosum Entoloma Horak

myceniformis Melanoleuca Sing.

mycenoides Gloiocephala Sing.

mycoderma Candida (Rees) Lodder \&

Kreger Van Rij.

mycophilum Physarum

myocopron Microphoma Speg.

my'oschili Microsphaera Neger

myosotis Naematoloma (Fr.) A. H

Smith.

myrceugenellae Phaeomarasmius

Sing.

myrceugeniae Brefeldiela Syd.

myrceugeniae Disculina Kcissl.

myriantha Asterina Syd

myrtacearum Napicladium Spcg.

myrticola Depazea Klotzch. myrticola Phyllosticta Sacc.

myrticola Sphaerella Speg.

myrtinum Pilidium Dur. \& Mont.

myxacioides Pholiota Sing.

myxoduracinus Cortinarius Horak

myzodendri Microsphaera Speer. \& Oehrens

nana Galerina (Petri) Kühner

nanus Pluteus (Pers. ex Fr.) Kummer

napobrassicae Phoma Rost.

nasellae Puccinia Arth. \& Holw.

nassauviae Uredo Vestergr.

natalensis Diplodia Pole-Evans

natalis Pholiota Sing.

naucinus Leucoagaricus (Lepiota)

(Fr.) Sing.

naumanniana Puccinia (Magn.) Diet. naumanniana Uropyxis Magn. navarinum Aecidium Jorst.

nebularum Agaricus Sing. necator Uncinula (Schw.) Burr.

necatrix Dematophora (Rosellinia)

Hartig

necatrix Rosellinia (Hartig) Berl.

necopinatum Entoloma Horak

negeriana Asterina Syd.

negeriana Meliola Syd.

negeriana Phyllachora P. Henn.

negeriana Parodiella Syd.

negeriana Pleuroplacosphaeria ( $\mathrm{P}$.

Genn.) Syd.

negeriana Puccinia Diet.

negeriana Teichosporella Sacc. \&

Syd.

negerianum Aecidium Diet.

negerianum Cenangium P. Henn.

negerianum Caeoma Dict.

negerianum Epiclinium Sacc. \& Syd.

negerianum Dimerosporium P. Henn.

negerianum Hysterium P. Henn. \&

Lindau.

negerianum Ustilago Dict.

neglecta Leptocladia

neglectu Pleurage (Hansen) Cl.

Moreau

neglecta Puccinia Magn

nematospora Allantophoma Kreb.

neoformans Cryptococcus (Sanfelice) Vuillemin

nerviphilus Uromyces (Grogn.) Hotson

nicotianae Debaryomyces Giovanozzi

nidulans Aspergillus (Eidam) Wint. 
nidulans Emericella

niger Aspergillus Van Tiegh.

nigra Comatricha (Pers.) Schrdet.

nigra Hohenbuehelia (Schw.) Sing.

nigrescens Nematelia Mont.

nigrescens Stemonites Rex:

nigricans Clavaria Lloyd

nigricans Rhizopus Ehrd.

nigripes Didymium (Lk.)Fr.

nigripes Marasmiellus (Schw.) Sing.

nigrita Anthracophyllum (Lév.)

Kalchbr.

nigritum Geoglossum (Fr.) Cke.

nigrohirtula Scutellinia (Svrck) Le Gal

nigrolineatus Pluteus Murr.

nigrum Epicoccum Lk.

nitellinus Cortinarius Moser

nitidus Arachnomyces

nitidus Polyporus Pers.

nitens Rhodophyllus (Velen,) Kühn, et Romagn.

nitschkei Cryptovalsa Fckl.

nivalis Scutellinia (Boud.) Le Gal

niveum Byssocaulon Mont.

niveum Cyclaneusma (Pers. ex. Fr.)

Di Cosmo. Peredo \& Minter

niveum Diderma (Rost.) Mc Br.

niveum Fusurium S. Sm.

niveum Lachnea Karst.

niveum Hydnum Pers.

niveum Sporotrichum Allesch. \& $\mathrm{P}$. Henn.

niveus Camarophyllus (Fr.) Wünsche nodulosporum Lyophyllum Sing. nordenskjöldii Uromyces Diet. nothofagi Elasmomyces Horak nothofagi Pluteus Horak nothofagi Uncinula Thast. nothofagii Leptothyrium Spcg. nothofagineus Marasmiellus Sing. nothomyrciae Marasmius Sing. nothomyrciae Mycena Sing. nothomyrciae Psathyrella Sing. novazelandiae Armillariella Stev. novisima Mycena (Speg.) Sing. novum Entoloma Horak nucleata Exidia (Schw.) Burt. nuda Lepista (Tricholoma, Agaricus)

(Bull. ex Fr.) Cke.

nuda Puccinia Ell. \& Everh. nuda Ustilago (Jensen.) Kell. et Swing. nudipes Hebeloma (Fr.) Kalch. nummularia Sphaeria Dec. nutans Physarum Pers.

obliqua Ovularia (Cke.) Oud. obliquus Irpex (Scharad.) Fr. obovata Peronospora Bon. obscura Puccinia (Schrad.) Fr. obscureolivellus Cortinarius Moser obscuroarmeniacus Cortiñarius. Moser

obtusa Physalospora (Schw.) Cooke obtusiceps Mycena Speg. occultum Entoloma Horak octayensis Hygrocybe Sing. ochracea Nectria Fr. ochracea Sphaeria Grev. ochraceocoeruleus Cortinarius Moser ochraceolamellatus Cortinarius Moser.

ochraceorobustum Tricholoma Sing. ochraceum Helotium Boud.

ochraceum Hydnum Pers. ochroolivellus Cortinarius Moser oedipus Pholiota (Cke.) Orton oehrensiana Entomophthora Aruta Carrillo. \& Montealegre oenotherae Aecidium Mont. oenotherae Septoria West. ohiensis Geaster (Cke.) Lloyd ohiensis Laccaria (Mont.) Sing. oleae Hendersonulina Speg. oleaginum Cycloconium Cast. oleicola Calospora Speg. oleraceae Phoma Sacc.

oligosperma Sphaeropsis Speg. olivacea Reticularia (Ehr.) Fr. olivaceiceps Symocybe (Sing.) Sing. olivaceobubalina Dermocybe Moser olivaceum Dimerium Syd. oliveoicterina Dermocybe Moser oliveoicterinus Cortinarius Moser olla Cyathus Batsch. ex Pers. ommiumsanctorum Mycena Sing. omniun-sanctorum Psilocybe Sing. omphalioides Melanomphatia (Sing.) Sing.

omphalioides Resupinatus Sing. omphalomorphus Agaricus Bert. \& Mont.

omphalophorus Marasmiellus Sing oniscus Omphalia (Fr.)Sacc. opuntiae Hendersonula Ell. \& Ev. orbiculare Helminthosporium Lév oreophila Corymelia (Speg.) Starb. oreophila Septoria Sacc.
ornithogaliHeterosporium Klotzsch orobi Uromyces (Pers.) Lév. orobi Uredo Schum. orthosporum Rhynchosporium Caldwell oryzae Aspergillus (Ahlburg) Cohn oryzae Rhizopus (Fr.) Lind. osornensis Marasmiellus Sing. osornensis Pluteus Horak ostreatus Pleurotus (Jacq.) Fr. ourisiae Puccinia Diet. \& Neger ovata Stemonitis Pers. ovata Trichosphaeria Butin ovina Lasiosphaeria Ces. \& De Not. ovina Sphaeria Pers.

ovisporum Phy'sarum G. Lister oxulicum Penicillium Currie. \& Thom oxalidis Ustilago Ell. \& Tracy oxyascum Lophodermium Speg. oxycanthae Podosphaera (DC.) D'By. oxyssorum Fusarium Schlecht pacificus Marasmius Sing. pachyderma Calvatia (Pk.) Lloyd pachydermica Bovista Speg. pachyloma Hypoxylon (Lèv.) Mont. pachynemeus Cortinarius Moser pachytheca Sphaerella Speg. pallida Descolea Horak pallicla Sphaeria Pers. pallidilamellatus Cortinarius Moser pallidissima Puccinia Speg. pamparum Ustilago Speg. pampeana Clavaria Speg. pampeana Puccinia Speg. pampeamus Agaricus Speg. pampeanus Coccomyces Speg panueoloides Bolbitius P. Henn. panattoniana Marssonina (Bert.) Neger panicea Badhamia (Fr.) Rostaf. panici-urvilleani Uredo Diet, \& Neger pannicola Chrysosporium (Corda) van Oorschot \& Stalpers pannareoides Lecidea Nyl. pannorum Geomyces (Link) Sigler \& Carmichael

pannosa Antennaria Berk. pannosa Fumago (Berk.) Speg. pannosa Sphaerotheca (Wallr.) Lév. pannosum Triposporium (Berk) Speg. panuoides Paxillus (Fr. ex $\mathrm{Fr}$ ) Fr. papillata Sphaeria Schum. papillatum Entoloma (Bres.) Dennis 
papillatum Enerthenema (Pers.) Rost. papillionaceus Agaricus Bull. papudense Aecidium Jacks. et Holw. papulata Laschia Mont. paraboliciformmis Mycena Sing. paradoxa Heterobotrys Sacc. paradoxa Pleurocollybia Sing. paradoxus Cortinarius Moser \& Horak parakrusei Candida (Cast.\& Chalm.) Lang. \& Guerra

paralella Xylographa (Ach.) Fr. parapsilosis Candida (Ashf.) Lang, \& Talice

parasitica Peronospora Pers. parasitica Phytophthora Dastur. parilis Agaricus Fr.

parilis Clitocybe $\mathrm{Fr}$.

parvispora Entomophthora McLeod $\&$ Carl.

parvula Campylospora

pasitheae Aecidium Diet. \& Neger pasitheae Puccinia Diet. \& Neger patagonica Bleunoria Speg. patagonica Clavaria Speg. patugonica Clavulinopsis (Speg.) Corner patagonica Clitocybe (Speg.) Sacc. patagonica Eutypa Speg. patagonica Gibbera Rehm patagonica Hemimycena Sing. patagonica Hohenbuehelia Sing. patagonica Pleosphaeria Speg. patagonica Ramaria (Speg.)Corner patagonica Scutellinia Rehm. patagonica Sphaerella Speg. patagonica Sphaerospora Rehm patagonica Ustilago (Cook. \& Mass.)

Cif.

patagonica Zignoella Speg. patagonicus Agaricus Speg. patagonicum Anthostoma Rehm. patagonicus Camarophyllus Sing. patagonicus Melanotus Sing. patagonii Aecidlum Speg. pataguae Sphaerella Speg. patouillardii Coprinus Quél. paucicolor Cortinurius Horak paucigibba Inocybe Sing. paxulutus Boletus Bert. peckianus Uromyces Farlow pectinatus Geaster peladae Collybia Sing. peladue Cortinarius Moser peladae Galerina Sing. peladue Psilocybe Sing. pelagica Monodictys (Johnson) Jones pelagicum Dictyosporium (Linder) G.C Hughes pellaeicola Hyalopsora Arth. pelleeae Uredo Diet. et Neger pellicula Chaetophoma Sacc. \& Syd. pelliculosa Asterina Berk.

pelliculosa Candida Red. pencana Puccinia Syd. pencana Uredo Diet. \& Neger pencanus Uromyces (Diet. \& Neger) Arth. \& Holw.

penetrale Physarum Rex. penicillioides Flagellospora Ingold penzigi Limacinia Sacc. perannans Ustilago Rost. perennis Coltricia (L. ex.Fr.)Murrill perforans Puccinia Mont. pergracilis Omphalia Spcg. perincerta Puccinia Jacks. et Holw. perlatum Lycoperdon Pers. permagnificus Cortinarius Horak permutatum Entoloma Horak pernettyae Mycosphaerella (Speg.) Syd.

perniciosum Fusarium Heptt. perpusilla Teichospora Spcg. perpusillus Coprinus Speg. perrara Galerina Sing. perseae Trochila Speg. perseicola Entosordaria Speg. persicina Tuberculina (Dittm.) Sacc. persiciphila Rhabdospora Speg. persimilis Trichia Karst. personata Lepista (Fr. ex Fr.) Cke. (Agaricus, Tricholoma)

persooni Helotium Mont. pertesii Galactinia (Berk. \& Curt.) Le Gal.

pesundatum Tricholoma $\mathrm{Fr}$. petaloides Pleurotus Bull. petroselini Septoria Desm. peullensis Campanella Sing. peullensis Entyloma Lindq. peullensis Marasmiellus Sing. peullensis Symocybe Sing. peumi Coniothyrium Speg. phacelliphila Sphaerella Speg. phacidioides Sporonema Desm. phacochlora Cercospora Speg. phacolaenarum Paneolus (Fr.) Qućl. phalligera Holuenbuchelia (Mont.)

$$
\text { Sing. (Agaricus) }
$$

phalligerus Crepidotus Mont. phalloides amanita (Vaill. ex Fr.)Secr. phaseoli Uromyces (Reb.) Wint phaseolina Macrophomina (Tassi) Goid. phenolicus Cortinarius Moser philesiae Phoma Speg. philippiana Physalospora Wint. philippii Claviceps Rehm. philippii Aecidium Speg. philippii Puccinia Diet. \& Neger phlegmacioides Galerina Sing. phragnites Puccinia (Schum.) Koern. phyllachoroides Puccinia Speg. phyllogena Mycena (Pers.) Sing. piceae Ceratocystis (Munch.) Bakshi. pilgerodendri Appendiculella Butin pilgerodendri Chloroscypha Butin pilgerodendri Hypoderma Butin pilgerodendri Marasmius Sing. pilifera Ceratocystis ( $\mathrm{Fr}$.) C.Moreau pilosus Lasiobolus (Fr.) Sacc. pinastri Lophodermium (Schrad.ex. Hook) Chev. pinastri Sphaeropsis Ell. \& Curt. pinca Caliciopsis Peck.

pini-densiflorae Cercoseptoria (Hori \& Nambu.) Deighton

pini Dothistroma Hulb.

pinus Torulopsis Lodder. \& Kreger Van Rij

piptochacti Puccinia Dict. \& Neger

pirelloides Mucor Lendt.

piri Elsinoë (Wor.) Jenks.

piri Plectodiscella Jenks.

piricola Diplodia Speg.

pisi Ascochyta Lib. pisi Peronospora Syd.

pisi Septoria Wesid

pisi Uromyces Fckl.

pithyophila Sclerophoma (Corda)

Sacc.

placeae Puccinia Dict. et Neger

placentula Uromyces Mont.

planchoniana Entomophthora Cornu

planiuscula Uredo Mont.

plantaginis Ramularia EII. \& G.

Martin

platensis Agrocybe (Speg.) Sing.

pleurotus Clitocybe Sing.

plicatilis Coprinus (Curt.) Fr.

plumbaria Puccinia Peck.

plumbeum Dictydiacthalium

(Schum.) Rost. 
poae-sudeticae Puccinia Jorst. poarum Puccinia Niels. podanthi Eutypa Speg. podanthi Cryptosphaerella Speg. podanthi Phoma Speg. podanthi Septoria Speg. podocarpi Dasyscypha Gamundi podocarpi Hypoderma Butin podocarpi Keissleriella Butin polyadelphus Cortinarius Moser polycocca Grandinia Fr. polycocca Thelephora Mont. polycystidiosa Psathyrella Sing. polychaetopus Marasmius Sing. polygoni Erysiphe Dc.

polygoni Peronospora A. Fisch polygoni Puccinia Pers. polygoni Uromyces (Pers.) Fckl. polygoni-amphibii Puccinia Pers. polygoni-aviculariae Uromyces

(Pers.) Karst.

polygonorum Puccinia Schlecht polygonorum Septoria Desm. polygramma Mycena (Fr.) S. F. Gray polymorpha Pichia Klöcker polymorphum Lycoperdon Vitt. polyphaga Erysiphe Hammalwnd. pomi Phoma Pass. pomi Sphaerella Pass. populi Hadrotrichum Sacc. populi Sphaceloma Jenk. populina Melampsora (Jacq.) Lév. populina Perichaena Fr. populina Phyllosticta Sacc. porphyrizon Agaricus Orton portegnus Pleurotus Speg. portentosa Sphaeria Mont. portentosa Xylaria Mont. portentosum Entoloma Horak porteri Hypoxylon Speg. porteri Macrophoma Speg. porterii Xerotus Speg. portulacae Albugo (DC.) Kuntze portulacae Cystopus (DC.) Lév. posidoniae Halotia Hariot pozoae Uromyces Diet. \& Neger praecox Agrocybe (Pers. exFr.) Fayod praetervisa Peziza (Galactinia) Bres. prasiolae Guignardia (Wint.) Viala. \& Ravaz prasiolae Laestadia Wint. pratense Vascellum (Pers.) Kreis. pratensis Hygrophorus Pers. pratervisa Galerina Sing. pratiae Uromyces Speg. primulae Ramularia Thüm. privigna Pholiota (Speg.) Sing. privignus Agaricus Speg. probus Uromyces Arth. procera Armillariella (Speg.) Sing. procera Agrocybe Sing. procera Macrolepiota (Lepiota)

(Scop.ex Fr.) Sing. procerus Uromyces Lindq. prolifica Periconia Anastasiou prolixa Collybia (Horn. ex Fr.) Sing. proteosperma Pleospora Speg. proteus Hygrocybe Sing. proustiicola Phyllosticta Speg. proximella Laccaria Sing. prunastri Epitea DC. prunastri Puccinia DC. prunastri Uredo DC. pruni Puccinia Pers. pruni-spinosae Puccinia Pers. pruni-spinosae Tranzschelia (Fckl.) Tranzs. \& Liv.

prunorum Puccinia Lk. prunulus Clitopilus Scop. psammopodioides Cortinarius Horak pseudhypoxylon Rosellinia Speg. pseudochinensis Rhizopus pseudomurale Gerronema (Speg.) Sing.

pseudopachyloma Hypoxylon Speg. pseudoradicata Oudemansiella Moser

pseudorussula Agaricus Speg. pseudostromatica Teichospora Rehm pseudosylvestris Galactinia Gamundi. pseudotriumphans Cortinarius Moser. \& Horak pseudotropicalis Candida (Cast.) Bagal.

psittacina Hygrocybe (Fr.) Kummer psittacinum Physarum Dit.

psoraleae Diplodia (Cast.) Karst.\& Harriot

pterospora Martelia Horak pudorinus Cortinarius Horak pulchella Alatospora Marvanova pulchellaMalbranchea Sacc. \& Penzig pulchella Propolis Speg. pulcherrima Campoa Speg. pulcherrima Candida (Lind.) Windish pulchra Clavulinopsis (Pk.) Corner pulchra Selinia (Wint.) Karst. pulicaris Cheilaria Mont. pulveracea Rosellinia (Ehr.) Fckl. pulveraceus Cenangium Fr. pulverulente-rosea Clavaria Speg. pulvinulus Sphaeria Berk. pulla Patellaria Fr. pullulans Dematium Fr. pullulans Trichosporum (Lind. \& Lodder) Diddens pumanquense Entoloma (Rhodophyllus) (Sing.) Horak

pumanquensis Clavaria Lazo pumanquensis Cortinarius Moser pumanquensis Cystolepiota Sing. pumanquensis Hyrocybe Sing. pumanquensis Xeromphalina Lazo. punctata Puccinia Lk.

punctato-striatum Caeoma Diet. et Neger punctatus Uromyces Schroet. punctiformis Peziza Fr. punctiformis Trichopeziza (Fr.) Fckl.

punicea Arcyria Pers. populina Badhamia A. et G.Lister pura Mycena (Pers. ex Fr.) Quél. purpurascens Epicoccum Ehr. \& Schletch ( $=\boldsymbol{E}$. nigrum LinK ex Link)

purpurascens Uredo Diet. \& Neger purpuratus Gymnopilus (Cke. \& Mass.) Sing.

purpurea Cercospora Cke. purpurea Cordyceps Fr. purpurea Claviceps (Fr.) Tul. purpurea Puccinia Cke. purpureum Hysterangium Zeller \& Dodge purpureum Stereum Pers. purpureus Polyporus Fr. pusilla Bovistella Lloyd pusilla Dacryomitra Tul. pusilla Cudoniopsis Speg. pusilla Didymosphaeria Speg. pusilla Ovularia (Ung.) Sacc. pusillisporum Tricholoma Speg. pusillus Favolus Fr. pusillus Rhizomucor (Lindt) Schipper pusio Tremellodendropsis (B.) Crawford pusticola Didymosphaeria Speg. putidus Boletus Horak

puyae Anthostomella P. Henn. \& Lind. puyae Microthelia Speg. 
puyae Metasphaeria Speg.

puyae Phoma Speg.

puyae Pleospora Speg.

puyae Sphaerella Speg.

puyae Sirococcus Speg.

puyae Venturia Speg.

puyaecola Anthostomella P. Henn.\& Lind.

pycnospora Periconia Fr.

pygmaeus Cyathus Lloyd.

pyriformis Cribraria (Schrad.) Pers.

pyrina Venturia Aderh.

pyrinum Fusicladium (Lib.) Fckl.

pyxidata Omphalina (Bull. ex Fr.)

Quél.

pyxidatoides Hydropus Sing.

quadrifida Propolis Mont.

quadrifida Stictis Lév.

quadrifidum Arthroderma Dawson \& Gentles

quevinae Chillöella Syd.

quevinae Glomerella (Syd.) V. Arx. et Müller

quillensis Puccinia Diet. \& Neger

quilliermondi Candida (Cast.) Lang. \& Guerra

quillotensis Peziza Mont. quinchamalii Uromyces Neger quinteroensis Galerina Sing.

racemosus Mucor Fres.

racovitsae Sarcoscypha Bomm. et Rouss.

rachodes Lepiota (Scop.) Quél.

radiata Schmitzonia Fr.

radiata Stictis (L.) Pers.

radiatum Diderma (1.) Morgan

radiatus Coprinus Fr.

radicinum Stemphylium (Miers.

Drechs. et Eddy) Meerg.

ramealis Marasmius (Bull, ex Fr.)

Sing.

rancidus Cortinarius Moser

ranunculi Aecidium Schw.

recondita Puccinia Rob. ex Desm.

recurvatus Camarophyllus (Peck)

Murr.

reductoaffinis Symocybe Sing.

reductum Entoloma Horak

reessii Gymmoascus Baran.

reessii Uncinocarpus Sigler \& Orr

reflexa Phlebia Berk.

reflexum Stereum (Bull.) Sacc.

rehmiana Ellisiodothis (Theis.\& Syd.)

Theis. et Syd. reicheana Gibbera (P. Henn.) Müller $\&$ Sanwal.

reicheana Montugnella $\mathrm{P}$. Henn.

reichei Cyttaria $\mathrm{P}$. Henn.

reichei Puccinia Diel. \& Neger

reichei Uromyces Diet.

reiliana Sphacelotheca (Kühn.) P.

Clinton

reniformis Endogone Bres.

reniformis Xylaria Starb.

repanda Pseudopeziza (Fr.) Karst.

repanda Peziza (Pers.) Fr.

repensActinomucor Schostakovitsch

resedens Descolea (Cke. et Mass) Sing.

resinosus Polyporus (Schrad.) Fr.

reticulospora Rosellinia Greis. \&

Greis Dengler Fr.

retirugis Paneolus $\mathrm{Fr}$.

rhabarvarinus Fomes Berk.

rhacodes Macrolepiota (Vitt.) Sing.

rhaphithamni Phyllosticta Speg.

rhaphitnamni Didymellina Keissl.

rheana Sarcoscypha Fckl.

rhenana Aleuria Fckl.

rhicnophilus Thelephora Lév.

rhizogena Patellaria Monj.

rhododendri Exobasidium Cramer

rhododendri Pestalotia (Sacc.) Guba

rhododendricola Phyllosticta Brun.

rhodostacheos Sphaerella Speg.

rhodostacheos Zignoella Speg

ribis Botryosphaeria (Tode) Gross. \& Dugg.

ribis Puccinia DC.

ribis Septoria Desm.

rickenii Conocybe (Schaeff.) Kühner

rigidula Ovularia Del.

rigidulum Stereum Speg.

rimosus Fomes (Berk.) Cke.

ripartitoides Entoloma Horak

roberstsii Cordyceps Hook

robertiani Stigmatea Fr.

robineae Pleospora (Lib.) Hoehn

robinsoniae Trematosphaeria Keissl.

robinsoniae Uredo Keissl.

robinsonii Antennaria Mont. \& Bert.

roridum Myrothecium Tode

rosaceus Agaricus Nees

rosae Alternaria

rosae Actinonema (Lib.) Fr.

rosae Diplocarpon Wolf

rosae Puccini DC.

rosarum Sphaceloma (PASS.) Jenk.

rosea Anguillospora Descals rosea Cystolepiota Sing.

rosea Hygrocybe Murr.

rosea Mycogone Link,

rosea Niptera Mont.

rosea Thelephora Pers.

rosei Saccharomyces(Gill.) Lodder.

\& Kreger van Rij

rosella Humidicutis (Speg.) Sing.

rosella Tubercularia Speg.

roseum Cephalothecium Cda.

roseum Fusarium (LK.) SM. \&

Hans.

roseum Gliocladium (Ld.) Barnier

roseum Sporotrichum Pers.

roseum Trichothecium Link. ex Fr.

rouxi Saccharomyces Boutroux

rubella Mycena Quél.

ruber Hymenogaster Harkn.

rubescens Cytospora $\mathrm{Fr}$.

rubescens Rhizopogon Tul,

rubi Fusarium Wint.

rubi Cylindrosporium Ell. \& Morgan

rubi Microthyrium Niessl

rubicola Cordelia Speg.

rubicola Entosordaria Speg.

rubicola Leptothyrium Speg.

rubicola Hypospila Speg.

rubiginosa Hymenochaete (Schrad.)

Lév.

rubiginosum Hypoxylon (Pers.) Fr.

rubiginosus Stereum Fr.

rubigo-vera Puccinia DC.

rubigo-vera Uredo DC.

rubra Exidia Bomm. \& Rouss.

rubra Rhodotorula (Demme) Lodder

rubra Torrubiella Path. \& Lagh.

rubricola Sphaeria Fr.

rubricosum Mastigosporium (Dearn. \& Barth.) Nannf.

rubricosum Hypoxylon Fr.

rubrobasalis Cortinarius Moser \& Horak

rubrum Trichophyton (Castellani)

Ota

ruderum Agaricus Bert. \& Mont.

ruderum Cortinarius Bert. et Mont.

rufa Hypocrea (Pers.)Fr.

rufo-fulva Thelephora Mont.

rufoflavus Polyporus Berk. \& Curt.

rufu-fulvum Corticium Fr.

rufula Tryblidiella (Spreng.) Sacc.

rufulum Rhytidlysterium (Spreng.)

Speg.

rugosa Peziza Lév. 
rugosa Thelephora Fr.

rugosum StereumFr.

rugulosus Marasmiellus (Berk. \&

Curt.) Sing.

rumicis Uromyces (Schum.) Wint. sabouraudi Trichophyton Blanchard

(=T. tonsurans Malmsten)

saccatum Geastrum (Geaster) Fr.

saccharinus Coprinus Romagn, in

Kühner. \& Romagn.

sagittariae Puccinia Rabh.

salicinum Capnodium Mont.

saligna Tympanis Tode

sapinea Sphaeropsis (Fr.) Dyko \&

Sutton

sambucina Auricularia Mart.

sambucinum Fusarium Fckl.

sanctae-crucis Caeoma Espinosa

sanguinea Nectria (Bol.) Fr.

sanguineus Polyporus L.

santiaguina Phyllosticta Speg.

santiaguinum Camarosporulum Speg.

saponariae Sorospodium Rudolphi

sarcinaeforme Stemphylium (Cav.)

Wiltsh.

sarcoides Coryne (Jacq.) Tul. (Bulgaria, Tremella)

sarmienti Cortinarius Speg.

sarmienti Stereum Speg.

saxegothaeae Dimerosporium $\mathrm{P}$.

Henn.

saxegothaeae Pezomella Syd.

saxegothaeae Phomopsis Syd.

saxegothaeae Pistillaria Sing.

saxegotheae Mycena Sing.

scabra Dothidothia Syd.

scalpturatum Tricholoma Fr.

scandens Helicomyces Morg.

scansilis Polyporus Fr.

scalaroides Diplocladiella

sciophana Hygrocybe (Fr.) Karst.

scirpi Fusarium Lamb. et Fautr.

scitula Nectria Syd.

sciurodes Cortinarius Moser \&Horak

sclerotiorum Sclerotinia (Lib.) D'By.

scolecinus Cortinarius Moser

scopiformis Xylaria Mont.

scoriadea Antennaria Berk.

scoriadea Chaetophoma Speg.

scoriadea Limacinia (Berk.) Keissl.

scottii Candida Diddens \& Lodder

scrobiculatus Lactarius (Scop. ex Fr.)

Fr.

scutellata Scutellinia (Peziza) (L.ex
Fr.) Lamb. scutellatus Uromyces (Schrank.) Lév. scyphoides Omphalia Fr. schachtii Peronospora Fckl. schizoxyla Omphalia Fr. schleidenii Peronospora Ung. schoenleinii Licea Johow schoenleinii Trichophyton (Lebert)

Langeron \& Milchevicht schroeteriana Erinella Sacc. \& Syd. secalis Rhynchosporium (Oud.) J.J Davis

seditiosum Lophodermium Minter, Staley \& Miller

segetum Uredo Pers.

segetum Ustilago (Bull,) Ditt.

selenospermum Anthracoderma Speg.

selenospora Endomycopsis (Nads. \& Krauss) Dekk.

semiglobata Stropharia (Agaricus)

(Batsch. ex Fr.) Quél.

semiglobatus Cortinarius Moser semiimersus Hypoxylon Nits. semilanceata Psilocybe (Fr.) Kummer semiorbicularis Naucoria Bull. semipellucida Dermocybe Moser. seneccionis-acanthifolii Aecidium Diet.

senecionis Coleosporium (Pers.) Fr. senecionis Ustilago Diet. \& Neger senex Fomes (Nees. \& Mont.) Cke. (Polyporus)

senex Phellinus Sing.

sepedonium Corynascus (Emmons) von.Arx

septembris Pholiota Sing.

septica Fuligo (Aethalium) (Lk.) Gmel.

septicus Agaricus Fr

septospora Cytosporina Dorog. sepulchralis Anellaria (Berk.) Sing. sequoiae Cercospora Ell. \& Evech.

serenus Seriomyces Hcinem. (Agari cus, Pscudobaeospora)

seriale Coniothecium Dur. \& Mont. sericeochraceus Cortinarius Moser sericeus Rhodophyllus (Bull. ex Fr.) Quél.

serpens Merulius Pers.

serpens Hypoxylon (Pers.) $\mathrm{Fr}$.

serpens Sphaeria Pers.

serpula Hemitrichia (Scop.) Rost. serratus Ctenomyces Eidam. setariae Puccinia Diet. \& Nolw.

setigerum Tetracladium (Grove)

Ingold

setipes Pistillaria Grev.

setosum Chaetomium Wint.

setulosa Fumago Lév.

sexdecemsporus Coprotus ( $\mathrm{Cr}$. et

Cr.) Kimbr. \& Korf.

sheareri Pharcidia Arn.

shedonnardi Puccinia Kell. \& Swingl.

sherardiana Puccinia Koern

siccans Helminthosporium Dresch.

( = Drechslera siccans (Drechsker) Shoem.

sierrae Psilocybe Sing.

sigmoidea Laboulbenia Speg.

silenes Septoria Ell. \& Mart.

silenicola Septoria Ell. \& Mart.

siliginea Conocybe (Fr.) Kühner

silphii Uromyces (Burr.) Arth.

simocyboides Galerina Sing.

simplex Diderma (Schroet.) List.

simplex Puccinia (Koern. Erikss.) \& P. Henn.

sinapizans Hebeloma (Paulet. ex Fr.)

Gillet

singeri Underwoodia Gamundi \&

Horak

sinuosum Angioridium Grev.

sinuosum Hebeloma Fr.

sinuosum Physarum (Bull.)Weinm.

ex Fr.

striatulus Cortinarius Speg.

sisyrinchii Puccinia Mont.

sisyrinchii Uromyces Mont.

stilboidea Cyphella Speg.

skottsbergii Cyttaria Palm.

skottsbergii Nectria Wesse

sneyderi Termitaria Thaxt.

sodale Entoloma (Kühn. \& Romagn.)

Horak

soderstromii Hyndnangium Lagh.

soluni Aecilium Mont.

solani Cephalothecium Ell, \& Mont.

solani Botryobasidium (Prill. \& Del.)

Donk

solani Candida Lodder \& Neger van $\mathrm{Rij}$

solani Alternaria Sorauer

solani Fusarium (Mart.) Appl. \& Wollenw,

solani Helminthosporium Dur. \& Mont.

soluni Macrosporium Ell. \& Mart. 
solani Thecaphora Var. solanii Rhizoctonia Kühn. solariae Uromyces Diet. solenia Peziza Bert. solidaginis Puccinia Pk. solidaginis-microglossae Puccinia Diet.

solitarie Uredo Diet. et Neger solivae Cystopus (Schroet.) Sacc. sonchi-arvensis Coleosporium (Pers.) Lév.

sordaria Sphaeria Fr. sordida Lepista (Fr.) Sing. sordidum Tricholoma $\mathrm{Fr}$. sordulentus Polyporus Mont. sorghi Cintractia (Sorok) de T. sorghi Puccinia Schw. sotae Marasmiellus Sing. spadiceo-atra Peziza Mont. sparrei Armillariella Sing. spathularia Dacryopinax (Schw.) Mart.

speciosa Volvariella (Fr.exFr.) Sing. (Volvaria)

spectabilis Gymnopilus (Fr.) A.H Smith

speeae Aecidium Gäum. spegazzinianus Pluteus Sing. spegazzinii Hohenbuehelia Sing. ex Sing.

spegazzinii Ustilago Hirsch speireoides Phaeomarasmius Sing. sphaeralceoides Puccinia Cumm. sphaeriodes Dothidea Fr. sphaerodermus Marasmius Speg. sphaerogena Ustilago Burr. sphaerosperma Entomophthora Fres. sphaerostigmatis Puccinia Diet \& Neger

sphagnorum Casaresia

sphinctrinus Paneolus (Fr.) Quél. spiculosa Sphaeria Pers. spinaciae Peronospora Lambert. spinicola Lasmeniella Syd. spiniferum Septobasidium Burt. spinulosa Lamprospora Seaver spiralis Sphaerotheca Neger. splendens Enteridium (Morgan) Mc $\mathrm{Br}$.

splendens Cribraria (Schrad.) Pers. splendens Stemonitis Rost.

splendens Tricladium splitgerberi Flavioporellus (Berk.) Murr. spongiosa Mucilago (Leyss.) Morg. sporoloides Uromyces Cumm. sporoboli Uromyces Ell. \& Ever spumarioides Diderma $\mathrm{Fr}$. spumosa Pholiota (Fr.) Sing. squamiger Cortinarius Moser squamosopunctus Pluteus Horak squamosum Tylostoma (Gmel.) Fr. squamosus Agaricus Pers.

squamosus Polyporus (Huds.) Fr. squamulosum Didymium (Alb. \& Schw.) Fr. squarrulosum Pachylepyrium Sing. stagmina Tubaria Fr. steinmanniac Puccinia Diet. \& Neger stella Omphalia Bomm. \& Rouss. stellariae Septoria Rob. \& Desm. stellata Clavatospora (Ingold \& Cox) S. Nilss. ex Marvan. \& S. Nilss. stellatoidea Candida (Jones \& Martin) Lang, \& Guerra. stellatus Dacryomyces Nees stellatus Sphtaerobolus Tode stelligerum Tripospermum Speg. stenandrii Puccinia Dict. \& Neger stercorea Cheilymenia Boud. stercorea Lachnea (Pers.) Sacc. stercorea Peziza Pers.

stercoreus Cyathus (Schw.) de Toni stercolurius Agaricus Fr.

stevennii Battarrea (Lib.) Fr.

stictica Asterina

stictica Xylographa

sticticum Sphaeronaema Berk.

stipatum Hydnum $\mathrm{Fr}$.

stipitata Hemitrichia (Mass.) $\mathrm{Mc} \mathrm{Br}$.

stipticoides Panus Speg.

stolonifer Mucor Elir.

stolonifer Rhizopus ( $\mathrm{Fr}$.) Lind.

stolpiana Cumminsiella (Diet. \& Neger.) Baxter

stolpiana Puccinia (Magn.) Dict. \& Neger

stolpiana Uredo Magn.

straminipes Physarum List.

straminis Puccinia Koern

strangulatu Hygrocybe (Orton) Sing.

striatella Hygrocybe Sing.

striatipes Conocybe (Speg.) Sing.

striatulus Resupinatus (Fr.) Murr.

striatus Cyathus (Huds.) Hoffm.

striatus Uromyces Schroct

striatus-Medicaginis Uromyces

striiformis Puccinia West. striiformis Ustilago (West.) Niessl striimarginata Melanoleuca Metrod stromaticum Coniosporium Cda. stromaticum Melanconyum Cda. stypticoides Marusmiellus (Speg.)

Sing.

subalbidum Thaxterogaster Smith. subandina Psathyrella Sing. subaphaerosporuum Corticium Listch subaurantiaca Ramaria Corner. subcinnabarina Nectria (Tode) Fr. subconicus Cortinarius Litsch. subcoprophila Psilocybe (Breitz.) Sacc.

subcorticalis Rhizomorpha Pers. subcorticium Phragmidium (Shrank) Wint.

subflamans Pholiota (Speg.) Sacc. (Agaricus)

subfuscum Tylostoma White subglobosus Debaryomyces (Zach.) Lodder \& Kreger van Rij subglutinosa Mycena Sing. subleteromorpha Hygrocybe Sing. subluybrida Collybia Sing. sublyygrophanoides Clitocybe Sing subinocyboides Cortinarius Moser sublateritium Naematoloma (Fr.)

Karst.

subleptoloma Clitocybe Sing. sublimbata Anthostomella (Dur.\& Mont.) Speg. sublimbata Sphaeria Dur. \& Mont. sublineata Torrubiella Petch.

sublitoralis Leucoagaricus (Kühner \& Horak Sing. submarginatus Pluteus Horak submastrucatus Pleurotus P. Henn. submersus Heliscus subnitens Puccinia Diet. subolivaceum Helminthosporium Speg. subordinaria Phoma Desm. subprona Psathyrella Sing. subrenispermum Coprinus Sing. substrictior Entoloma (Rhodophyllus) (Sing.) Horak.

subterranea Spongospora (Wallr.) Lagh.

subtilissima Lachnellula (Cooke) Dennis

subulifera Mycena Sing. subvillosa Cyphella P. Henn. 
succosella Peziza (LeGal\&Romagn.) Moser \& Gams (Galactinia)

sulfurea Malbranchea (Miehe) Cooney \& Emerson

sulphureus Arachnomyces Masse \& Salmon

sulphureus Polyporus (Bull.) Fr. suppariger Cortinarius Moser t Horak surculum Lentaria (Bk.) Corner sylvestris Peziza (Galactinia) (Boud.) Moser

symphiti-bromorum Puccinia Muell. symphyostemi Phoma Speg.

syntherismae Sorosporium (Peck) Farl.

syringae Ascochyta Bres. syringae Oidium Blumer tabacina Chaetoporus Mont. tabacina Hymenochaete (Sow.) Lév. tabacina Helvella Mont. tabacina Peronospora Adams. tabacina Thelephora Fr. tabularis Mycosphaerella Syd. tacnensis Cortinarius Speg. taedium Entoloma Horak talcahuanense Leptothyrium Speg. talcahuanensis Sphaeronaema Speg. taraxaci Puccinia (Reb.) Plowr. taraxaci Synchytrium D'By. \& Wor. tarnensis Clitocybula (Speg.) Sing. (Cantharellula)

tehuelche Agaricus Speg. tehuelches Omphalia Speg. temucensis Uredo Jacks. \& Holw. tenebricosum Entoloma Horak tenebrosum Aecidium Jacks. \& Holw. tener Xerotus Berk. \& Br. tenera Galera Scheff. tenerrima Conocybe Sing. tenerrima Mycena (Berk.) Sacc. tenerum Scleroderma Berk. \& Curt. tentacula Clavatospora

tenuipes Setchelliogaster (Setch.) Pouzar tenuipes Xeromphalina (Schw,) Smith tenuis Alternaria Nees

tenuissima Alternaria (Fr.) Wiltsh tenuissima Hymenochaete Berk. tenuissima Sphaeria Schw. tenuissima Xylaria (Zipp. \&Lév.) Fr.

tepae Candida Grinbergs tephrophyllus Cortinarius Moser teres Helminthosporium Sacc. termophila Myceliopthora (Apinis) van Oorschot

termophile Chaetomium La Touche termophilum Acremonium W. Gams \& Lacey

termophilum Scytalidium (Cooney \& Emmerson) Austwick

termophilus Dactylomyces Sopp terrestre Trichophyton Durie \& Frey terrestre Tricladium Ingold terrestris Lemonniera Tubaki terrestris Pyrenochaeta (Hans.) Goerenz. terrestris Thelephora Ehr. terreum Tricholoma (Schaeff. ex Fr.) Kummer (Agaricus)

terreus Aphanoascus terreus Aspergillus Thom. tessariae Puccinia (Speg.) Diet. tessariae Uredo Speg. testaceum Diderma (Schrad.) Pers. tetracladia Articulospora Ingold tetrasphaerophora Mycena Sing. tetraspora Laccaria Sing. texensis Scutellinia (Berk. \& Curt.) Le Gal

thalassica Puccinia Speg.

thalictri-poarum Puccinia Fisch \& May

thaxteri Hyndnangium Zeller \& Dodge

thaxteri Puziza Cash

thaxteri Nothojafnea (Cash) Gamundi thaxteriana Entomophthora (Petch) Hall. \& Bcll.

theleboloides Cheilymenia (Alb. \& Schw. ex Fr.) Boud.

theleboloides Cilaria Alb. \& Schw. theleboloides Scutellinia (Alb. ex Sch. ex Fr.) Kunze

thelephorum Hydnum Lév. thermarum Aecidium Diet. \& Neger thlaspi-Glaucophilii Puccinia P. Henn.

thureti Saprolegnia D'By. thymicola Mycena Velen.

tigrensis Crepidotus (Speg.) Sacc. tilcariensis Psathyrella Sing. titubans Helotium Mont. togularis Cortinarius Horak tonsurans Trichophyton Malmsten torcuata Halosphaeris Kohlmeyer trabea Trumetes Otth.

trachyspermus Cortinarius Moser tragopogonis Albugo Gray transformans Puccinia Diet. trechispora Stemonitis (Berk.) Mc Br. tremellinus Corticium Berk. trevelyani Diderma (Grev.) Fr. trevoa Camarosporulum (Speg.)

\section{Steyaert} trevoa Chrysalidopsis Speg. trevoa Melanomma Speg. trevoa Hendersonulina Speg. trevoa Massariella Speg. trevoa Pestalotia Speg. trevoa Phoma Speg. trevoae Diplodia Speg. trevoae Leptosphaeria Speg. trevoae Phomatospora Speg. trevoae Pleospora Speg. trevoicola Pleospora Speg. triangulospora Ceratocystis (Butin) Upadhyay tricapillata Gyoerffyella Marvan. tridactyla Podosphaera (Wallr.)D'By. trifolii Pseudoplea (Pers.) Rost. trifolii Polythrincium Kunze trifolii Pseudopeziza Bernh. trifolii Uromyces (Hedw.) Lév. trifolii-fallens Uromyces (Desm.) Arth.

trifolii-megalanthi Aecidium Diet \& Neger

trifolii-megalanthi Uromyces (Diet \& Neger) Jacks. \& Holw.

trifoliorum Peronospora D'By.

trifoliorum Sclerotinia Ericks.

trimorpha Puccinia Neger

triplex Geastrum (Geaster) Jungl.

triplotricha Mycena Sing.

triptilii Puccinia Mont.

triscupus Clathrus Fr.

triteleiae Uromyces Diet. \& Neger

tritici Septoria Desm.

tritici Urocystis Koern.

tritici Tilletia (Beijerk.) Wint. tritici Ustilago (Pers.) Rost.

triticina Puccinia Erikss.

triviale Corticium Speg.

trochus Melanomma Penz. \& Sacc.

tropica Corynelia (Awers. \& Rab.) Starb.

tropicalis Candida (Cast.) Berhout tropicum Chrysosporium Carmichel truncatus Saccobolus Vel. truncorum Coprinus (Scheff.) Fr. tubiforme Aecidium Diet. \& Neger 


\begin{tabular}{|c|c|c|}
\hline $\begin{array}{l}\text { iolacea Peziza Pers. } \\
\text { iolacea Ustilago (Pers.) Fckl. } \\
\text { iolaceum Phragmidium (Schults) } \\
\text { Wint. } \\
\text { iolaceus Cortinarius Fr. (Agaricus) } \\
\text { iolaceus Polyporus Fr. } \\
\text { iolae Cercospora Sacc. } \\
\text { iolae Puccinia (Schum.) DC. } \\
\text { iolarum Puccinia Lk. } \\
\text { irgineus Cortinarius Horak \&Moser } \\
\text { irgultorum Hypoderma DC. } \\
\text { iride Chloridium Link. } \\
\text { irideMicroglossum (Pers.exFr.) Gill. } \\
\text { iride Physarum (Bull.) Pers. } \\
\text { iride Trichoderma Pers. } \\
\text { iscida Galerina (Peck) Smith.\& Sing. } \\
\text { iscidulus Hydropus Sing. } \\
\text { iscosa Agrocybe Sing. } \\
\text { iscosa Calocera Fr. } \\
\text { iscovenetus Cortinarius Horak } \\
\text { italbae Pleospora (De N.) Berlese }\end{array}$ & $\begin{array}{l}\text { vitelliana Exidia Lév. } \\
\text { vitellina Diatrype Mont. } \\
\text { vitellina Hirneola Lév. } \\
\text { vitellinum Gloeosoma (Lév.) Bres. } \\
\quad \text { (Cyphella, Aleurodiscus) } \\
\text { vitellinus Bolbitius (Pers. ex Fr.) Fr. } \\
\text { viticola Phomopsis Sacc. } \\
\text { vitis Alternaria Cav. } \\
\text { vitis Pleospora Catt. } \\
\text { voqui Phoma Speg. } \\
\text { vulgare Crucibulum Tul. } \\
\text { vulgare Leptostroma Fr. } \\
\text { vulgaris Botrytis } \mathrm{Fr} . \\
\text { vulgaris Epigymatia Fr. } \\
\text { vulgaris Eriosphaeria Speg. } \\
\text { vulgaris Pholiota } \text { (Fr) Kummer } \\
\text { vulgaris Polyporus } \mathrm{Fr} \text {. } \\
\text { vulgaris Scleroderma Horn. } \\
\text { vulgaris Tubercularia Tode. } \\
\text { werdermanii Puccinia Syd. } \\
\text { wilkommii Fusarium Lind. }\end{array}$ & 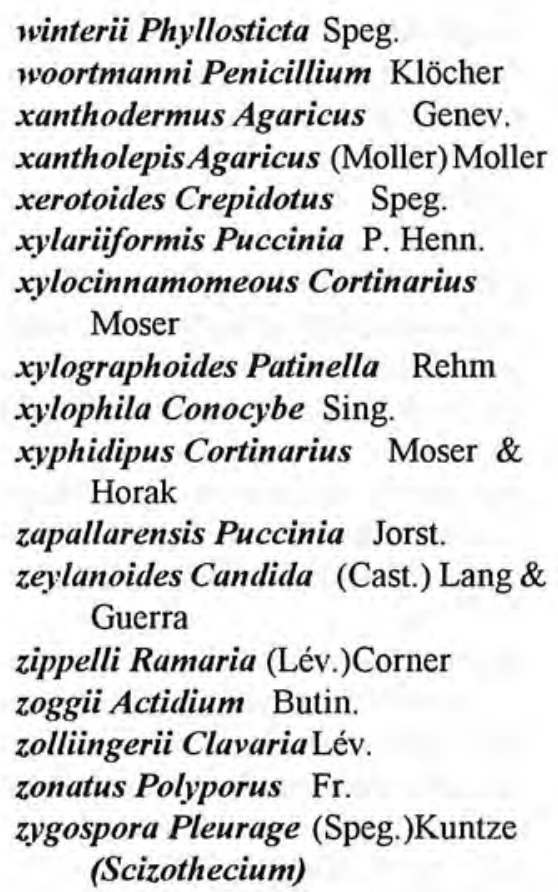 \\
\hline
\end{tabular}

